\title{
A critical displacement approach for predicting structural instability
}

\author{
Eugenio Oñate*, William T. Matias ${ }^{1}$ \\ E.T.S. Ingenieros de Caminos, Canales y Puertos, Universidad Politécnica de Cataluña, c/Gran Capitán s/n, 08034 Barcelona, \\ Spain
}

Received 31 March 1995

\begin{abstract}
A new technique for predicting structural instability points using the finite element method is presented. The approach is based on the estimation of the critical displacement pattern by writing an approximation of the tangent stiffness singularity condition at the instability point. The critical load is subsequently computed by using a secant load-displacement relationship. Details of this procedure are given together with explicit forms of the secant stiffness matrix for finite element analysis of solids and trusses. The accuracy and effectiveness of the method are clearly shown in a number of examples of two- and three-dimensional bar structures.
\end{abstract}

\section{Introduction}

The numerical detection and location of bifurcation and limit points, herein denoted generically as critical points, have received considerable attention in the computational solid and structural community. Indeed, loss of stability and bifurcation are common phenomena in non-linear solid and structural mechanics. Typical examples range from classical problems such as the buckling of rods, plates and shell structures, to diffuse necking bifurcation problems, including the formation of localized shear bands, in elastic-plastic solids.

The numerical methods proposed for computation of critical points can be grouped into two categories, namely, indirect and direct methods. With indirect methods the encounter of the critical point is judged with the help of a detecting parameter while the equilibrium path is being traced in a load incremental manner up to the vicinity of the critical point [1-6]. Typical examples of detecting parameters are the determinant or the smallest eigenvalue of the tangent stiffness matrix. The occurrence of a critical point is signified by the vanishing of both such parameters [7-16].

In direct methods the condition for occurrence of a critical point is included in the system of equations to be solved. The solution of the set of extended equations yields directly the position of the critical point and its associated eigenmode together with the load parameter.

Once a critical point has been found a path-switching algorithm has to be subsequently applied to follow the deformation of the structure along the possible bifurcation paths $[17,18]$. A review of direct and indirect methods and path switching strategies can be found in [19].

In this paper a new approach for detecting critical points is proposed. The method is based on the

\footnotetext{
* Corresponding author.

${ }^{1}$ Lecturer on leave from Department of Civil Engineering, University of Brasilia, Brazil.
} 
prediction of the critical displacement pattern. This is found by writing the tangent stiffness singularity condition at the critical point using a predicted perturbation of the last converged displacement field. The problem can be posed as a non-linear eigenvalue one which can be simply linearized to provide an accurate estimate of the displacement pattern at the critical point. The critical load can be subsequently computed using a secant load-displacement stiffness relationship. The type of critical point (i.e. limit or bifurcation point) can be simply detected by computing the eigenvector corresponding to the (approximate) tangent stiffness matrix at the critical point.

The ideas presented in this paper are an extension of the work reported by the authors in the development of non-linear solution procedures based on the secant stiffness matrix [20-22]. The concept of the critical displacement method was firstly sketched in the work of Kroplin et al. [23-25]. To the authors' knowledge, this idea has not been exploited or even further developed towards practical use.

The lay-out of the paper is as follows. First some simple concepts of elastic stability analysis are given together with the basic equations of geometrically non-linear solid mechanics. Then, the derivation of the secant stiffness matrix, which is an essential ingredient of the approach proposed, is described for three-dimensional solids. The critical displacement methodology is then presented in some detail and some alternatives to enhance its computational efficiency are discussed. The accuracy of the new approach is validated with examples of application to the detection of limit and bifurcation points in two- and three-dimensional truss structures.

\section{Basic concepts of elastic stability}
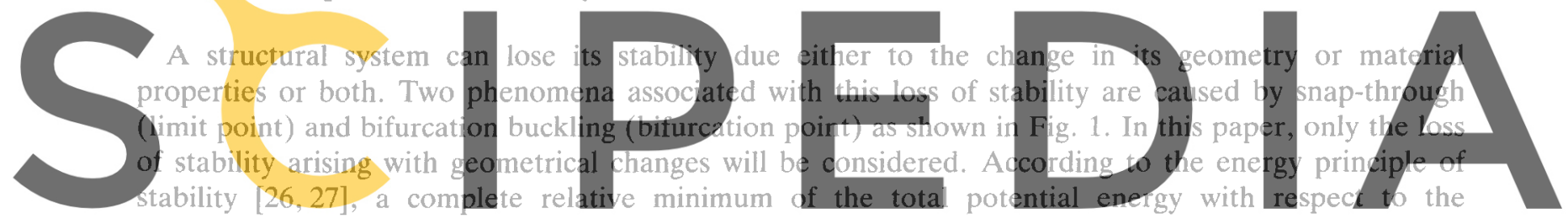

generalized coordinates is necessary and sufficient for the stability of an equilibrium state of the

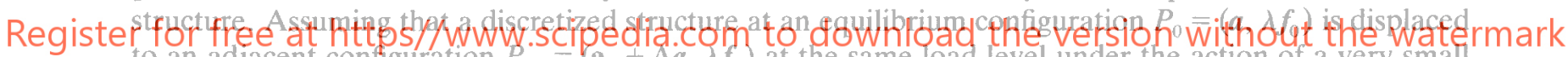
to an adjacent configuration $P_{\mathrm{ad}}=\left(\mathrm{a}_{0}+\Delta a, \lambda f_{0}\right)$ at the same load level under the action of a very small

perturbation $\Delta a$, then the change in potential energy can be expressed as

$$
\Delta \Pi=\Pi_{\mathrm{ad}}-\Pi_{0}=\Pi_{, i} \Delta a_{i}+\frac{1}{2} I_{, i j} \Delta a_{i} \Delta a_{j}+\frac{1}{6} \Pi_{, i j k} \Delta a_{i} \Delta a_{j} \Delta a_{k}+\cdots \quad i, j, k=1, \ldots, N
$$

where $\Pi_{\text {ad }}, \Pi_{0}$ are the potential energy at states $P_{\text {ad }}$ and $P_{0}$, respectively, $(\cdot)_{, i}$ denotes the partial

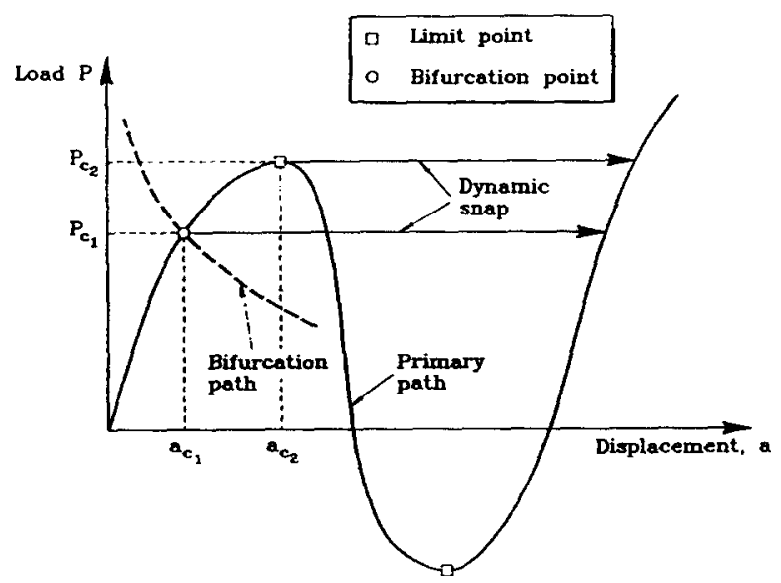

Fig. 1. Critical points: limit and bifurcation points. 
derivative with respect to the displacement increment $\Delta a_{i}, a$ is the vector of nodal displacements, $\lambda$ and $f_{0}$ are the dimensionless loading parameter and the vector of equivalent nodal forces, respectively, and $N$ is the total number of nodes. Summation convention is used in (1) for repeated indices.

Since $P_{0}$ is an equilibrium state then $\Pi_{i}=0$. Also with infinitesimally small perturbation the contribution from third-order terms and greater can be neglected (typically only second-and third-order terms appear in (1) in most structural problems $[26,27])$. Hence, the stability condition can be simply written as

$$
\frac{1}{2} \Pi_{, i j} \Delta a_{i} \Delta a_{j}=\frac{1}{2} \Delta a^{\mathrm{T}} K_{\mathrm{T}} \Delta a>0
$$

where $\boldsymbol{K}_{\mathrm{T}}=\Pi_{i j}$ is the tangent stiffness matrix. The stability of an cquilibrium configuration can be judged by investigating the positive definiteness of the tangent stiffness matrix. The equilibrium path is stable at $P_{0}$ if the determinant of $\boldsymbol{K}_{\mathrm{T}}$, denoted hereafter $\left|\boldsymbol{K}_{\mathrm{T}}\right|$, is greater than zero, where for $\left|\boldsymbol{K}_{\mathrm{T}}\right|<0$ the equilibrium path is unstable. At a critical point where $\Delta \Pi=0$ and $K$ is semi-positive definite, there exist some non-zero vector $\phi$ satisfying the following relationship

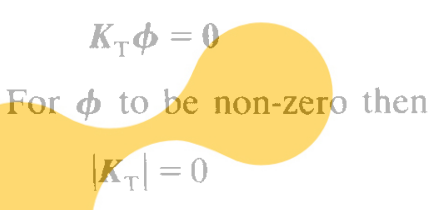

This condition is equivalent to the solution of the following eigenvalue problem

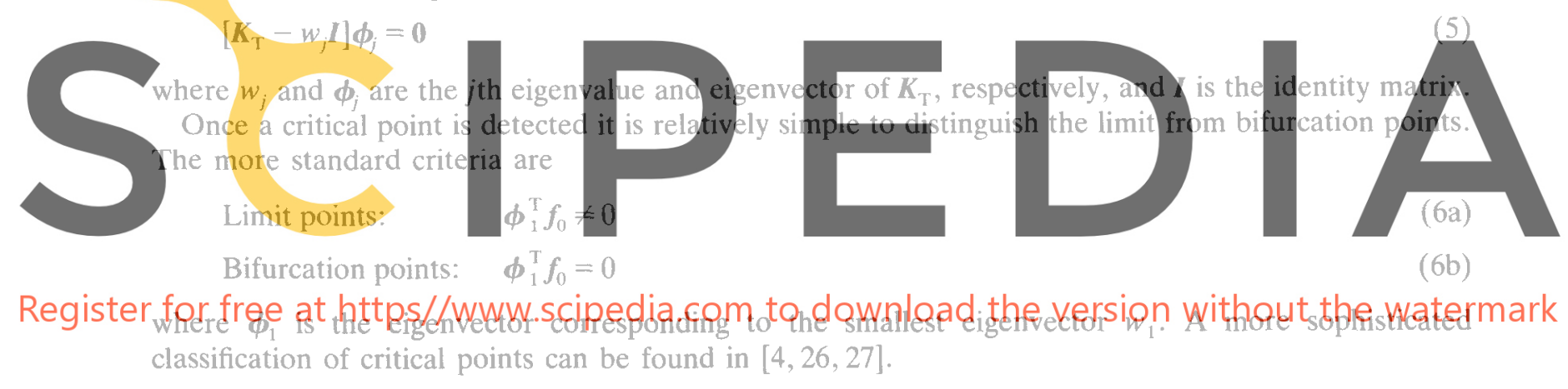

\section{Basic ideas of the critical displacement method}

The approach proposed here is based on the assumption that the critical displacement vector $\boldsymbol{a}_{\mathrm{c}}$ can be written as

$$
a_{\mathrm{c}}=a_{0}+\Delta a_{\mathrm{c}}
$$

where $a_{0}$ is the displacement vector at the known equilibrium configuration $P_{0}$. Vector $\Delta a_{\mathrm{c}}$ is now assumed to be of the form $\Delta a_{\mathrm{c}}=\lambda \boldsymbol{\phi}$ where $\phi$ is an estimation of the critical displacement increment pattern. The simplest choice $\phi=a_{0}$ can be chosen as shown in the examples given in the paper.

The displacement field (7) can be used to write the tangent stiffness singularity condition at the critical point (Eq. (4)) as the following non-linear eigenvalue problem

$$
\left|\boldsymbol{K}_{\mathrm{T}}+\lambda \boldsymbol{K}_{1}(\boldsymbol{\phi})+\lambda^{2} \boldsymbol{K}_{2}\left(\boldsymbol{\phi}^{2}\right)\right|=0
$$

where $\boldsymbol{K}_{\mathrm{T}}$ is the tangent stiffness matrix at the known equilibrium configuration $P_{0}$ and $\boldsymbol{K}_{1}$ and $\boldsymbol{K}_{2}$ are linear and quadratic functions of the predicted critical displacement increment pattern, respectively. Eq. (8) can be simplified by neglecting the quadratic terms. Once the minimum eigenvalue $\lambda$ is found, the critical displacement vector is obtained as $\boldsymbol{a}_{\mathrm{c}}=\boldsymbol{a}_{0}+\lambda \boldsymbol{\phi}$.

The value of the critical load vector $f_{c}$ can be subsequently computed from the secant loaddisplacement relationship, i.e. 


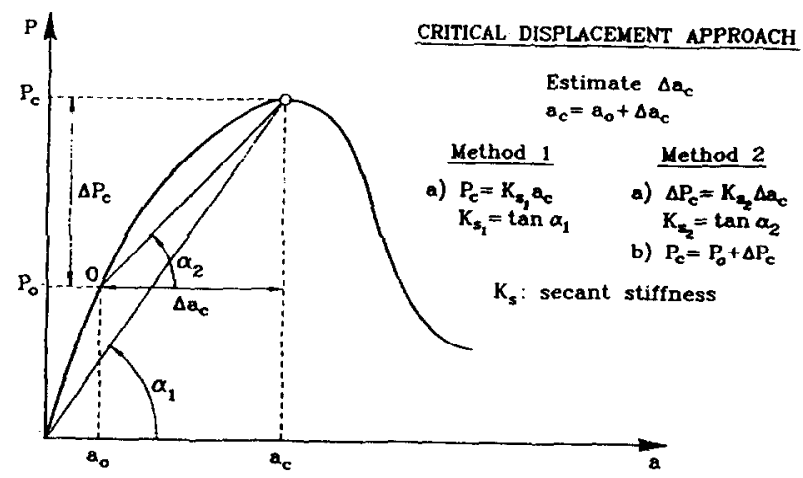

Fig. 2. Schematic representation of the critical displacement approach.

$$
f_{c}=\boldsymbol{K}_{\mathrm{S}}\left(a_{\mathrm{c}}^{2}\right) a_{\mathrm{c}}
$$

where $\boldsymbol{K}_{\mathrm{S}}$ is the secant stiffness matrix which has a quadratic dependence on the nodal displacements [23]. This process is schematically shown in Fig. 2.

This procedure has been proved to give very accurate predictions of both the critical displacement and critical load values even when the initial equilibrium configuration $P_{0}$ is taken to correspond with that given by an infinitessimal linear load-displacement relationship. Obviously, the accuracy improves when the initial displacement field $a_{0}$ approaches the critical value.
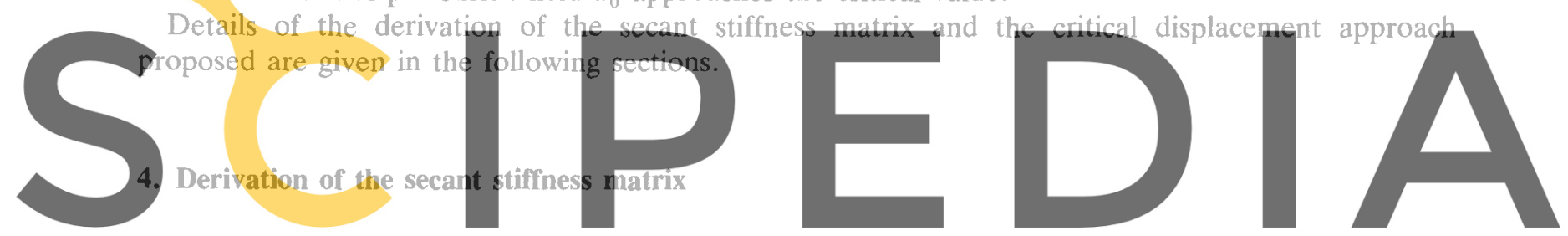

The potential of using the 'exact' form of the secant stiffness matrix for developing new solution

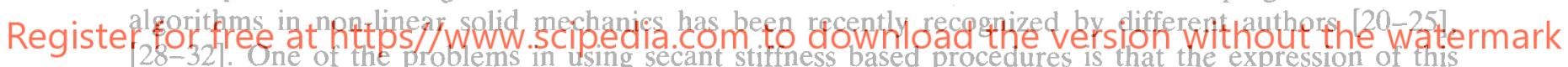
matrix is not unique and non-symmetrical forms are found unless a careful derivation is performed. Different symmetric expressions of the secant stiffness matrix have been obtained by several authors in the context of the finite element displacement method and a total Lagrangian description [33-39]. Alternative symmetric forms based on a mixed formulation were successfully derived and exploited by Kroplin and coworkers [23-25,30]. Recently, Oñate [22] has developed a general methodology for deriving the secant stiffness matrix for geometrically non-linear analysis of solids and trusses using a generalized Lagrangian description. This methodology will be followed in this paper and its basic ingredients are given next.

\subsection{Basic non-linear equations}

Let us consider a three-dimensional body with initial volume ${ }^{n} V$ in equilibrium at a known configuration ${ }^{t} V$ under body forces ${ }^{t} b$, surface loads ${ }^{t} t$ and point loads ' $p$. As usual, the superscript $t$ denotes a particular time or load level in dynamic or quasistatic analysis, respectively. When the external forces are incremented the body changes its configuration from ${ }^{t} V$ to ${ }^{t+\Delta t} V$. The coordinates of the body at each configuration are referred to the global Cartesian system $x_{1}, x_{2}, x_{3}$. The displacements at $t+\Delta t$ are (in vector form) [40]

$$
{ }^{i+\Delta t} \boldsymbol{u}={ }^{\prime} \boldsymbol{u}+\Delta \boldsymbol{u}
$$

where ${ }^{t} u$ are the known displacements at time (or load level) $t$ and $\Delta u$ are the sought displacement increments (see Fig. 3). 


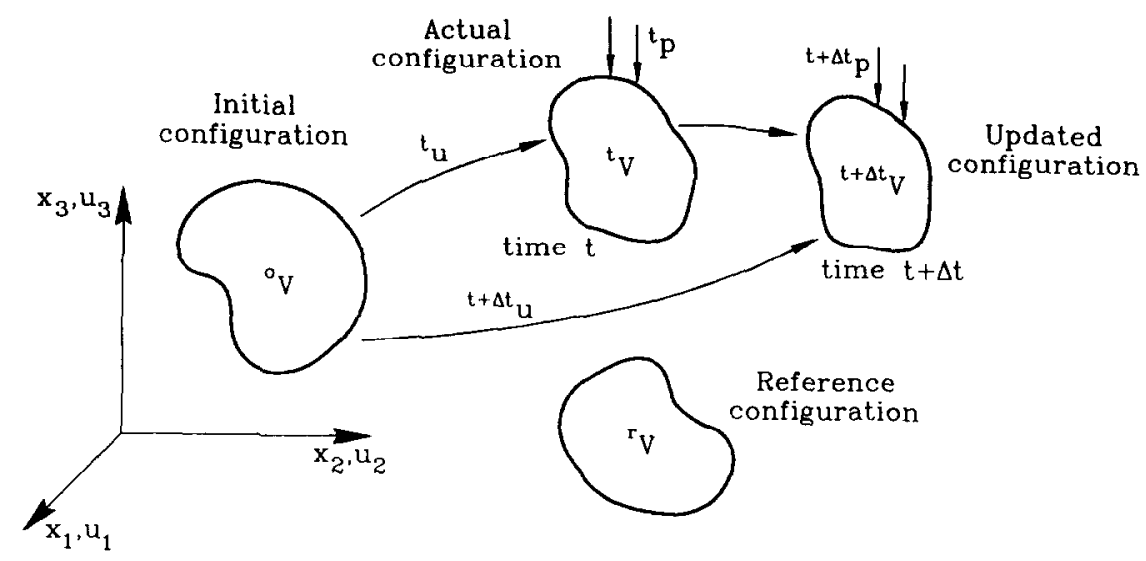

Fig. 3. Deformation of a body in a stationary coordinate system.

A generalized Lagrangian description will be used in which strains and stresses are referred to an intermediate reference configuration ${ }^{r} V$. (Fig. 3).

The strain tensor at $t+\Delta t$ referred to the configuration ${ }^{r} V$ can be written as

$$
{ }_{r}^{+\Delta t} \varepsilon=\frac{1}{2}\left({ }^{t+\Delta t}{ }_{r} u_{i, i}+{ }^{t+\Delta t}{ }_{r} u_{j, i}+{ }^{t+\Delta t}{ }_{r} u_{k, i}{ }^{t+\Delta t}{ }_{r} u_{k, i}\right)
$$
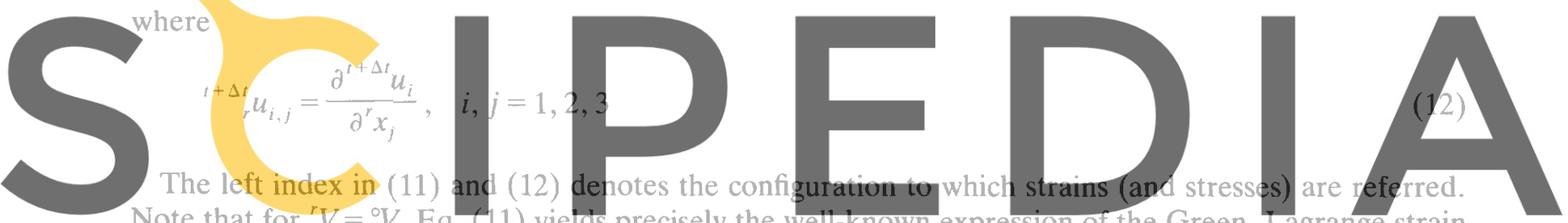

Note that for ${ }^{r} V={ }^{\circ} \mathrm{V}$, Eq. (11) yietas precisely the weli-known expression of the Green-Lagrange strain tensor in the total Lagrangian (TL) description. Also for ${ }^{r} V={ }^{t} V$ the expression of the linear part of the

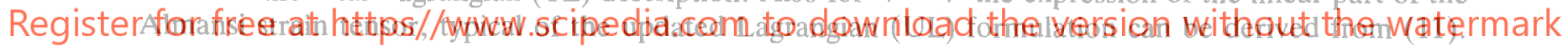

The strain increments are obtained as

$$
{ }_{r} \Delta \varepsilon_{i j}={ }^{+\Delta{ }_{r}} \varepsilon_{i j}-{ }_{r} \varepsilon_{i j}={ }_{r} e_{i j}+{ }_{r} \eta_{i j}
$$

where ${ }_{r} e_{i j}$ and ${ }_{r} \eta_{i j}$ are the first- and second-order strain increments. From (10) and (11) it can be obtained

$$
\begin{aligned}
& { }_{r} e_{i j}=\frac{1}{2}\left({ }_{r} \Delta u_{i, j}+{ }_{r} \Delta u_{j, i}+{ }_{,{ }_{t} u_{k, i} \Delta u_{k, j}+{ }_{,} \Delta u_{k, i}{ }_{r} u_{k, j}}\right)=0 \text { for }{ }^{r} V={ }^{t} V \\
& { }_{r} \eta_{i j}=\frac{1}{2}{ }_{r} \Delta u_{k, i}, \Delta u_{k, j}
\end{aligned}
$$

where

$$
{ }_{r} \Delta u_{i, j}=\frac{\partial\left(\Delta u_{i}\right)}{\partial^{r} x_{j}} \quad i, j=1,2,3
$$

Eqs. (14a) and (14b) are easily particularized for the TL and UL formulations simply by making $r=0$ and $r=t$, respectively. Note that the underlined terms in (14a) arc zcro in the UL formulation $\left({ }^{r} V={ }^{t} V\right)$.

For convenience we will write the first- and second-order strain increment vectors as

$$
\begin{aligned}
& { }_{r} \boldsymbol{e}=\left[\boldsymbol{L}_{0}+{ }_{r}^{t} L_{1}\left({ }_{r}^{t} g\right)\right], g \\
& { }_{r} \boldsymbol{\eta}=\frac{1}{2}{ }_{r} L_{1}\left({ }_{r} g\right)_{r} g
\end{aligned}
$$


In the above, ${ }_{r}^{t} \boldsymbol{g}$ and ${ }_{r} \boldsymbol{g}$ are displacement and displacement increment gradient vectors, respectively, $\boldsymbol{L}_{0}$ is a rectangular matrix containing ones and zeros and ${ }_{r}^{t} \boldsymbol{L}_{1}$ and, $\boldsymbol{L}_{1}$ are displacement and displacement increment dependent matrices, respectively. The form of all these matrices and vectors is given in Appendix A.

The virtual strains are defined as the first variation of the strains in the configuration ${ }^{t+\Delta t} V$. On the other hand, the displacements ${ }^{t} u_{i}$ can be considered as fixed during the deformation increment and thus $\delta{ }^{t} u_{i}=0$. Taking this into account we can write

$$
\delta^{t+\Delta t}{ }_{r} \varepsilon_{i j}=\delta{ }_{r} e_{i j}+\delta{ }_{r} \eta_{i j}
$$

where

$$
\begin{aligned}
& \delta_{r} e_{i j}=\frac{1}{2}\left(\delta_{r} \Delta u_{i, j}+\delta_{r} \Delta u_{j, i}+{ }_{r}^{t} u_{k, i} \delta_{r} \Delta u_{k, j}+\delta_{r} \Delta u_{k, i}{ }^{t} u_{k, j}\right) \\
& \delta_{r} \eta_{i j}=\frac{1}{2}\left(\delta, \Delta u_{k, i}, \Delta u_{k, j}+{ }_{r} \Delta u_{k, i} \delta, \Delta u_{k, j}\right)
\end{aligned}
$$

with

$$
\delta, \Delta u_{i, j}=\frac{\partial\left(\delta \Delta u_{i}\right)}{\partial^{r} x_{j}}, \quad i, j=1,2,3
$$

where $\delta \Delta u_{i}$ are the virtual displacement increments. Again the underlined terms in (19a) are zero in the

UL formulation. In matrix form we can write from (16) and (17)
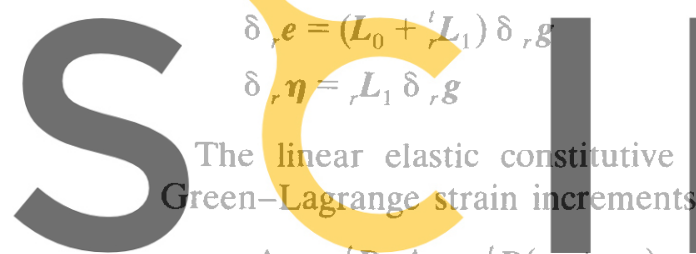

$r \Delta \sigma={ }_{r} D, \Delta \varepsilon={ }_{r}^{t} D(r e+r \eta)$
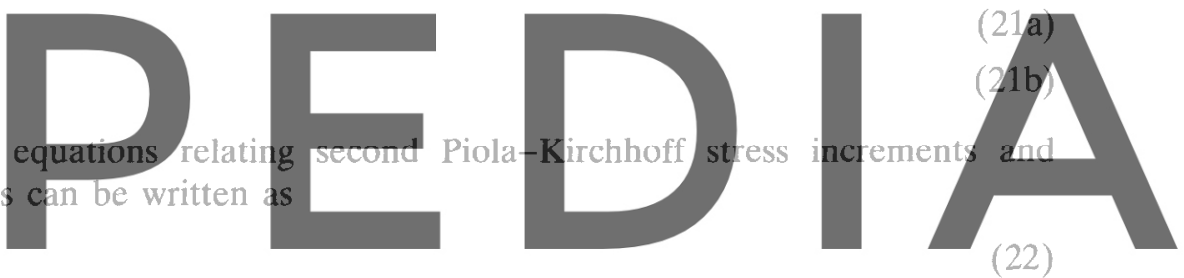

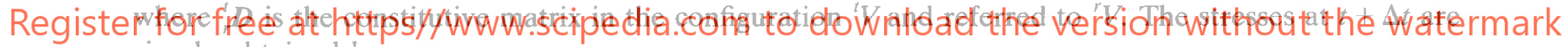
simply obtained by

$$
{ }_{r}^{+\Delta t} \sigma={ }_{r}^{t} \sigma+, \Delta \sigma
$$

Finally, the principle of virtual work (PVW) at ${ }^{t+\Delta t} V$ can be written in matrix form as

$$
\int_{r_{V}} \delta^{t+\Delta t}{ }_{r} \boldsymbol{\varepsilon}^{\mathrm{T}}{ }_{r}^{t+\Delta t} \boldsymbol{\sigma} \mathrm{d} V=\int_{r_{V}} \delta^{t+\Delta t} \boldsymbol{u}^{\mathrm{T} t+\Delta t} \boldsymbol{b} \mathrm{d} V
$$

where

$$
{ }^{t+\Delta t} \boldsymbol{b}=\left[{ }^{t+\Delta t} b_{1},{ }^{t+\Delta t} b_{2},{ }^{t+\Delta t} b_{3}\right]^{\mathrm{T}}
$$

For simplicity only body forces $b$ are assumed to act in (24a).

From Eqs. (10), (18), (23) and (24) and noting again that $\delta^{t} u=0$, Eq. (24a) can be rewritten as

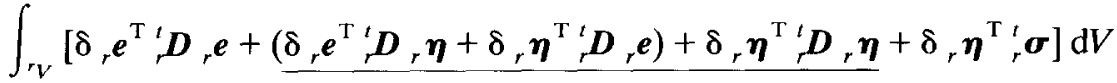

$$
\begin{aligned}
& =\int_{r_{V}} \delta \Delta u^{\mathrm{T} t+\Delta t} b \mathrm{~d} V-\int_{r_{V}} \delta, e^{\mathrm{T} t}{ }_{r} \sigma \mathrm{d} V
\end{aligned}
$$

Eq. (25) is the full incremental form of the PVW and it is also the basis for obtaining the incremental finite element equations. Note that the right-hand side of (25) is independent of the displacement increments and it will lead to the expression of the out of balance or residual forces after discretization. On the other hand, all the terms in the left-hand side are a function of the displacement increments. In particular, note that the underlined terms in (25) contain quadratic and cubic expressions of the 
displacement increments. The consideration of these terms is crucial for the derivation of the secant stiffness matrix. A linearization of Eq. (25) will neglect these terms, yielding the standard tangent stiffness matrix. The derivation of these two matrices for elasticity problems is presented in the following sections.

\subsection{Finite element discretization. Derivation of the secand stiffness matrix}

We will consider a discretization of a general solid in standard 3D isoparametric $C^{0}$ continuous finite elements with $n$ nodes and nodal shape functions $N^{k}(\xi, \eta, \zeta)$ defined in the natural coordinate system $\xi, \eta, \zeta$.

The displacement and displacement increment fields within each element are defined by the standard interpolations $[40,41]$

$$
{ }^{t} u=N^{t} a \text { and } \Delta u=N \Delta a
$$

where

$$
\begin{aligned}
& N=\left[N^{1}, N^{2}, \ldots, N^{n}\right] ; \quad N^{k}=N^{k} I_{3} \\
& { }^{t} a=\left\{\begin{array}{c}
{ }^{t} \boldsymbol{a}^{1} \\
\vdots \\
{ }^{t} \boldsymbol{a}^{n}
\end{array}\right\} ; \quad \Delta a=\left\{\begin{array}{c}
\Delta \boldsymbol{a}^{1} \\
\vdots \\
\Delta \boldsymbol{a}^{n}
\end{array}\right\} ; \quad \begin{array}{l}
{ }^{t} \boldsymbol{a}^{k}=\left[{ }^{t} u_{1}^{k},{ }^{t} u_{2}^{k},{ }^{t} u_{3}^{k}\right]^{\mathrm{T}} \\
\Delta \boldsymbol{a}^{k}=\left[\Delta u_{1}^{k}, \Delta u_{2}^{k}, \Delta u_{3}^{k}\right]^{\mathrm{T}}
\end{array}
\end{aligned}
$$

are the shape function matrices and the displacement and displacement increment vectors of the element and of a node $k$, respectively, and $I_{3}$ is the $3 \times 3$ unit matrix.

\section{Substitution of (28) int} in terms of the nodal disp
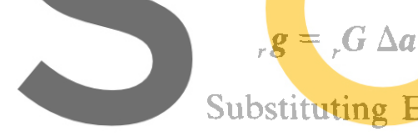

Substituting Eq. (28) into Eqs. (16) and (21) give
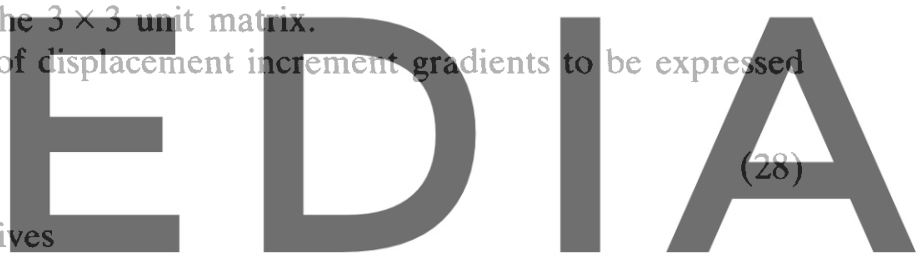

\section{${ }^{e}={ }^{\prime} B_{\mathrm{L}} \Delta a, \quad \delta, e={ }^{i} \boldsymbol{B}_{\mathrm{L}} \delta(\Delta a)$ \\ Register for free at https//www.scipedia.com to download the version without the watermark $\eta=\frac{1}{2}, B_{1} \Delta a, \quad \delta, \eta=, B_{1} \delta(\Delta a)$}

Matrix ${ }_{7}^{t} B_{\mathrm{L}}\left({ }^{t} a\right)$ can be split as

$$
{ }_{r}^{t} \boldsymbol{B}_{\mathrm{L}}\left({ }^{t} a\right)=, \boldsymbol{B}_{\mathrm{L}_{0}}+{ }^{t} \boldsymbol{B}_{\mathrm{L}_{1}}\left({ }^{t} a\right)
$$

where $\boldsymbol{B}_{\mathrm{L}_{0}}$ is the standard displacement independent matrix as derived from infinitesimal theory $[40,41]$ and ${ }_{1}^{\prime} B_{L_{1}}$ is the displacement-dependent part of the first-order strain increment matrix. This matrix vanishes in the case of the UL formulation.

The form of all above matrices for the case of 3D solids is given in Appendix A. Further details can be found in [22].

The incremental constitutive equations (22) can be written now in terms of the nodal displacement increments as

$$
{ }_{r} \Delta \boldsymbol{\sigma}={ }_{r}^{t} D\left[{ }_{r}^{t} B_{\mathrm{L}}+\frac{1}{2}, B_{1}\right] \Delta a
$$

Substituting Eqs. (29) and (31) into the PVW expression (25), the following relationship relating the total applied forces with the nodal displacement increments can be obtained

$$
{ }_{r}^{t} K_{\mathrm{S}}(\Delta a) \Delta a=-{ }^{t+\Delta t}{ }_{r} r
$$

In Eq. (32), ${ }_{r}^{i} r$ is the standard residual force vector which can be written for cach element with volume ${ }^{r} V^{(e)}$ as 


$$
{ }^{t+\Delta t}{ }_{r}{ }^{(e)}=\int_{r_{V}(e)}{ }_{r}^{t} B_{\mathrm{L}}^{\mathbf{T}}{ }_{r} \boldsymbol{\sigma} \mathrm{d} V-{ }^{t+\Delta t} \boldsymbol{f}^{(e)}
$$

with

$$
{ }^{i+\Delta t} f^{(e)}=\int_{v_{V}(e)} N^{t+\Delta t} b \mathrm{~d} V
$$

being the equivalent nodal force vector for the element, and ${ }_{r}^{t} K_{\mathrm{S}}$ is the incremental secant stiffness matrix which can be written as

$$
{ }_{r}^{t} K_{\mathrm{S}}(\Delta a)={ }_{i}^{i} K_{\mathrm{L}}+{ }_{,}^{\prime} K_{\mathrm{M}}(\Delta a)+{ }_{,}^{\prime} K_{\mathrm{N}}\left(\Delta a^{2}\right)+{ }_{,}^{t} K_{\sigma}
$$

where for each element

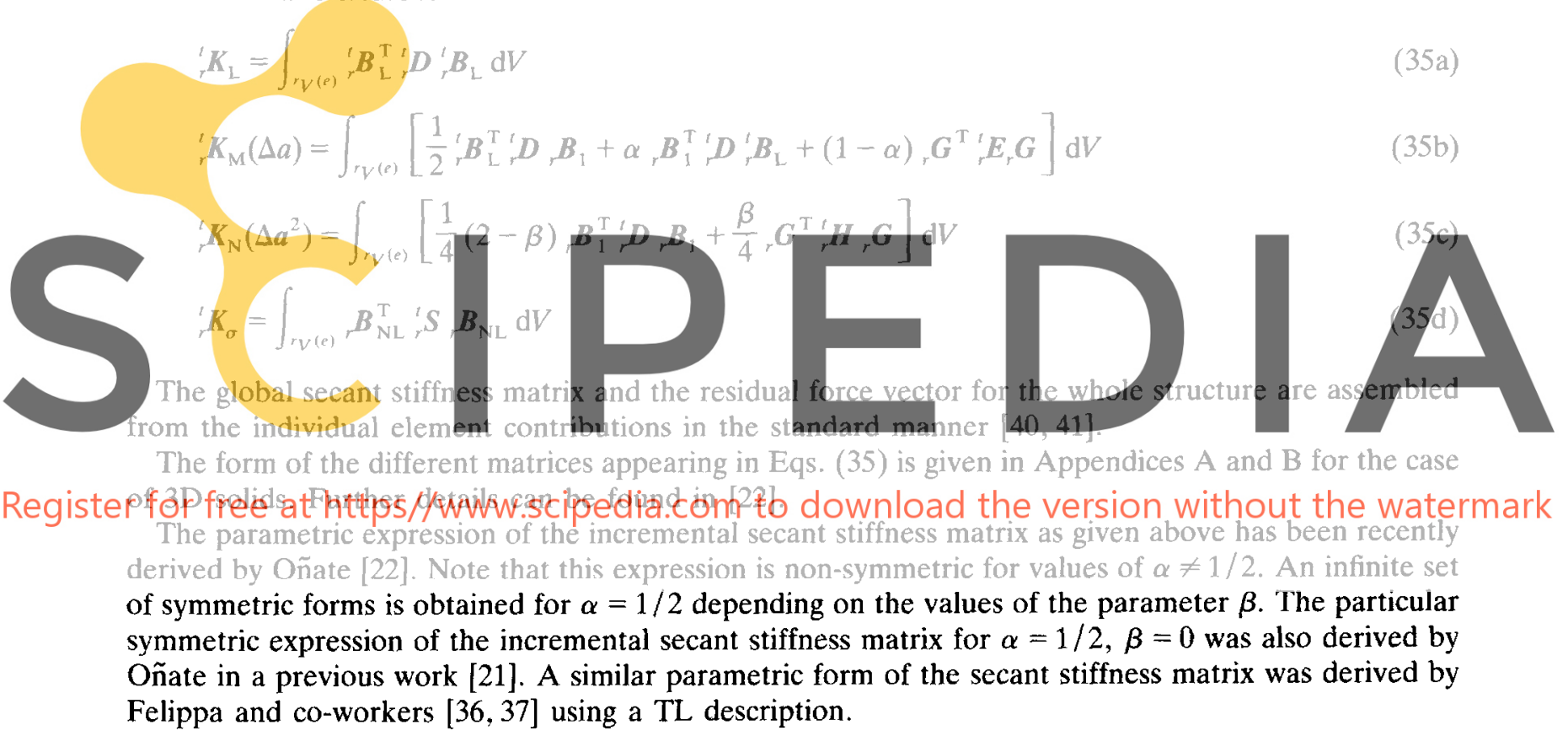

\subsection{Iterative solution techniques}

Eq. (32) can be used to solve for the new equilibrium configuration at $t+\Delta t$ by means of an incremental secant approach (Fig. 4a) giving

$$
\begin{aligned}
& \Delta a^{i}=-\left[{ }_{r}^{t} K_{\mathrm{S}}\left({ }^{t+\Delta t} a^{i}, \Delta a^{i-1}\right)\right]^{-1{ }^{i+\Delta t}}{ }_{r} r^{i} \\
& { }^{t+\Delta t} a^{i+1}={ }^{t+\Delta t} a^{i}+\Delta a^{i}
\end{aligned}
$$

with ${ }^{t+\Delta t} a^{0}=t a$ and $\Delta a^{-1}=\mathbf{0}$. Convergence of the iteration process is controlled by the satisfaction of an adequate norm in the nodal displacement increments or the residual force vector [40, 41].

A particular case of the above procedure corresponds to that with the residual force vector kept constant during the iterations. This can be simply interpreted as the satisfaction of the following incremental load-displacement relationship (Fig. 4b)

$$
{ }^{t} K_{\mathrm{S}}(\Delta a) \Delta a=\Delta f
$$

The iterative process reads now simply 


$$
r=P(a)-f
$$

a) Incremental secant approach b) Total secant approach (incremental loading)
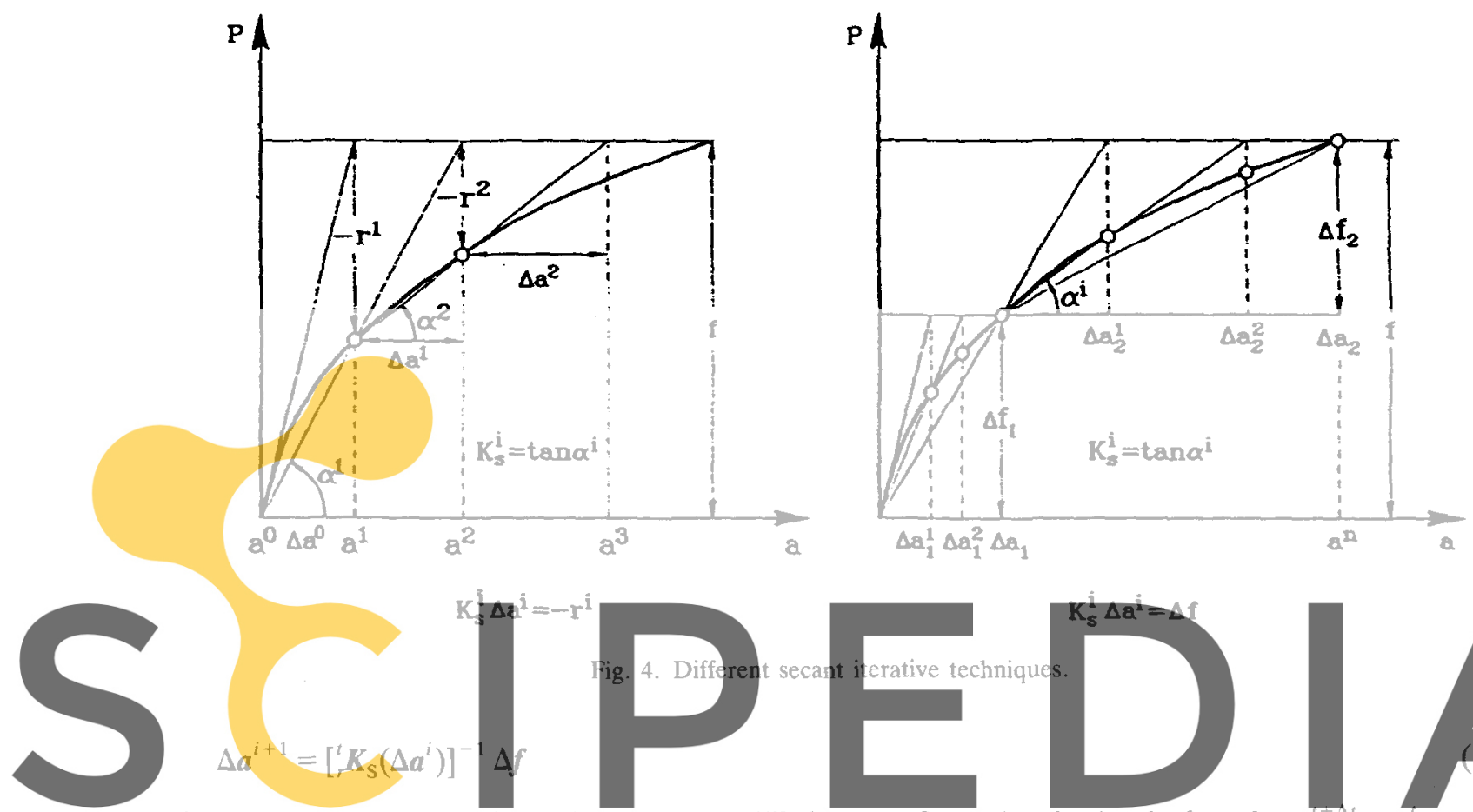

Once convergence is achieved the new equilibrium configuration is simply found as

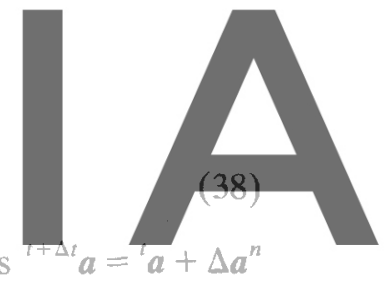

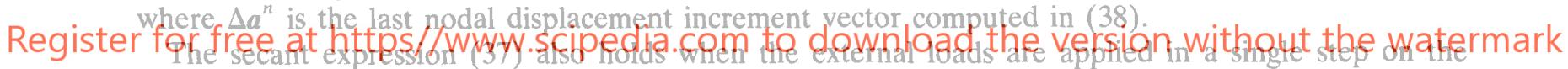

initial load-free configurations (i.e. $t=0$ ). Now the load and displacement increments in Eq. (37) are in

fact 'total' values and the same iterative process of Eq. (38) can be used to find the total displacement vector in a direct manner [22]. Note that in this case matrix ${ }_{r}^{t} \boldsymbol{K}_{\sigma}$ does not contribute to the secant stiffness matrix since the stresses are zero in the load-free configuration.

\subsection{Derivation of the tangent stiffness matrix}

The expression of the tangent stiffness matrix can be simply obtained as the limit of the incremental secant matrix when the values of the displacement increments tend to zero. Thus, from Eq. (34) we can write

$$
{ }_{r}^{t} \boldsymbol{K}_{\mathrm{T}}=\lim _{\Delta a \rightarrow 0}{ }^{t} \boldsymbol{K}_{\mathrm{S}}={ }_{r}^{t} \boldsymbol{K}_{\mathrm{L}}+{ }_{r}^{t} \boldsymbol{K}_{\sigma}
$$

Note that the resulting tangent stiffness matrix coincides with the standard expression obtained by linearizing the PVW in (25).

For subsequent purposes it is useful to rewrite matrix ${ }_{r}^{\prime} K_{\mathrm{L}}$ using (30) as

$$
{ }_{r}^{t} K_{\mathrm{L}}\left({ }^{t} a\right)={ }_{r}^{t} K_{\mathrm{L}_{0}}+{ }_{,}^{t} K_{\mathrm{L}_{1}}\left({ }^{t} a\right)
$$

where

$$
{ }_{r}^{t} \boldsymbol{K}_{\mathrm{L}_{0}}=\int_{r_{V}^{(e)}}, \boldsymbol{B}_{\mathrm{L}_{0} r}^{\mathrm{T}}{ }^{t} D, \boldsymbol{B}_{\mathrm{L}_{0}} \mathrm{~d} V
$$


is the standard stiffness matrix from infinitesimal elasticity theory and

$$
{ }_{r}^{t} \boldsymbol{K}_{\mathrm{L}_{1}}\left({ }^{\prime} \boldsymbol{a}\right)=\int_{V_{V^{(e)}}}\left({ }_{r} \boldsymbol{B}_{\mathrm{L}_{0},}^{\mathrm{T}}{ }_{r}^{t} \boldsymbol{D}_{r}^{t} \boldsymbol{B}_{\mathrm{L}_{1}}+{ }_{r}^{t} \boldsymbol{B}_{\mathrm{L}_{1}}^{\mathrm{T}}{ }_{r}^{t} \boldsymbol{D}_{r} \boldsymbol{B}_{\mathrm{L}_{0}}+{ }_{r}^{t} \boldsymbol{B}_{\mathrm{L}_{1}}^{\mathrm{T}}{ }_{r}^{t} \boldsymbol{D}_{r}^{t} \boldsymbol{B}_{\mathrm{L}_{1}}\right) \mathrm{d} V
$$

is the so-called initial displacement stiffness matrix [40,41]. An alternative expression of this matrix can be found in [22].

Note finally that the expression of the secant matrix (39) can be rewritten as

$$
{ }_{,}^{i} K_{\mathrm{S}}={ }_{,}^{i} \boldsymbol{K}_{\mathrm{T}}+{ }_{,}^{i} \boldsymbol{K}_{\mathrm{M}}+{ }_{,}^{i} \boldsymbol{K}_{\mathrm{N}}
$$

\section{Estimates for limit and bifurcation points. Critical displacement approach}

A useful application of the concept of a secant stiffness matrix is the estimation of the load level originating structural instability (i.e. limit or bifurcation points). These points are characterized by the singularity of the tangent stiffness matrix ${ }^{t}, K_{\mathrm{T}}$. The approach proposed here is based in the estimation of the critical displacement values giving singularity of ${ }_{r}^{t} \boldsymbol{K}_{\mathrm{T}}$, instead of those of forces as done in classical limit load theory. The secant stiffness relationship is then used to find the critical loading in terms of the critical displacement values in a straightforward manner. Details of this procedure are given next.

The process starts with the prediction of the displacement vector in the critical state as

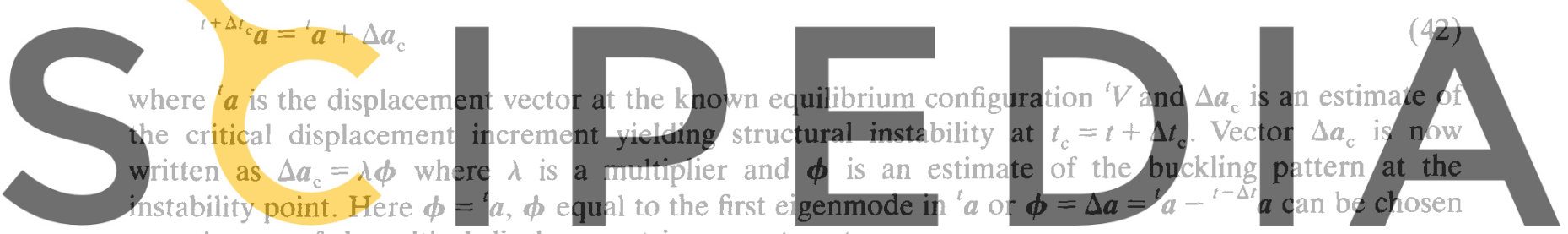

as estimates of the critical displacement increment vector.

Register Wit fhese assumptions the stress field at the critical point can be written, as (using Eqs. (22, 23, 31

$$
{ }^{i} \boldsymbol{\sigma}=\boldsymbol{\sigma} \boldsymbol{\sigma}+{ }_{r} \boldsymbol{D}\left[{ }_{r} \boldsymbol{B}_{L}\left({ }^{i} \boldsymbol{a}\right)+\frac{\lambda}{2}, \bar{B}_{1}(\boldsymbol{\phi})\right] \lambda \boldsymbol{\phi}=\boldsymbol{\sigma}+\lambda \boldsymbol{\sigma}_{1}+\lambda^{2} \boldsymbol{\sigma}_{2}
$$

where

$$
\begin{aligned}
& \boldsymbol{\sigma}_{1}={ }_{r}^{t} \boldsymbol{D}{ }_{r}^{t} \boldsymbol{B}_{\mathrm{L}}\left({ }^{t} \boldsymbol{a}\right) \boldsymbol{\phi} \\
& \boldsymbol{\sigma}_{2}=\frac{1}{2}{ }_{r}^{t} \boldsymbol{D}, \overline{\boldsymbol{B}_{1}}(\boldsymbol{\phi}) \boldsymbol{\phi}
\end{aligned}
$$

Substituting Eq. (42) into (30) allows to write the first-order strain matrix at the critical point as

$$
{ }_{r}^{i} \boldsymbol{B}_{\mathrm{L}}=,_{,} \boldsymbol{B}_{\mathrm{L}_{0}}+{ }_{r}^{t} \boldsymbol{B}_{\mathrm{L}_{1}}\left({ }^{t} \boldsymbol{a}\right)+\lambda, \overline{\boldsymbol{B}}_{1}(\boldsymbol{\phi})={ }_{r}^{t} \boldsymbol{B}_{\mathrm{L}}\left({ }^{t} \boldsymbol{a}\right)+\lambda, \overline{\boldsymbol{B}}_{1}(\boldsymbol{\phi})
$$

In Eqs. (43)-(44), ${ }_{,} \bar{B}_{1}(\phi)$ is obtained from the expression of ${ }_{1} B_{1}$ of Eq. (29) (see also Appendix A) simply substituting $\Delta a$ by the known predicted increment displacement pattern $\phi$. Note that when $\boldsymbol{\phi}={ }^{t} \boldsymbol{a}, \overline{\boldsymbol{B}}_{1}={ }_{r}^{t} \boldsymbol{B}_{L_{1}}$.

The tangent stiffness matrix can be written at the critical point taking into account Eqs. (39), (40), (43) and (44) as

$$
{ }_{i}^{t} \boldsymbol{K}_{\mathrm{T}}={ }_{r}^{t} \boldsymbol{K}_{\mathrm{T}}+\lambda\left({ }_{r}^{t} \boldsymbol{K}_{\mathrm{L}_{2}}+{ }_{i}^{t} \boldsymbol{K}_{\sigma_{1}}\right)+\lambda{ }^{2}\left({ }_{r}^{t} \boldsymbol{K}_{\mathrm{L}_{3}}+{ }_{r}^{t} \boldsymbol{K}_{\boldsymbol{\sigma}_{2}}\right)
$$

where ${ }_{r}^{\prime} \boldsymbol{K}_{\mathrm{T}}$ is the tangent stiffness matrix at the known equilibrium configuration ${ }^{i} V$ and 


$$
\begin{aligned}
{ }_{r}^{t} \boldsymbol{K}_{\mathrm{L}_{2}} & =\int_{r_{V^{(l)}}}\left[{ }_{r} \boldsymbol{B}_{\mathrm{L}}^{\mathrm{T}}{ }_{r}^{t} \boldsymbol{D}_{r} \overline{\boldsymbol{B}}_{1}+, \overline{\boldsymbol{B}}_{1}^{\mathrm{T}}{ }^{t} \boldsymbol{D}_{r}^{t} \boldsymbol{B}_{\mathrm{L}}\right] \mathrm{d} V \\
{ }_{r}^{t} \boldsymbol{K}_{\mathrm{L}_{3}} & =\int_{r_{V^{(l)}}}{ }_{r} \overline{\boldsymbol{B}}_{1}^{\mathrm{T}}{ }_{r}^{t} \boldsymbol{D}, \overline{\boldsymbol{B}}_{1} \mathrm{~d} V \\
{ }_{r}^{t} \boldsymbol{K}_{\boldsymbol{\sigma}_{1}} & =\int_{r_{V^{(l)}},} \boldsymbol{B}_{\mathrm{NL}}^{\mathrm{T}}{ }_{r}^{t} \boldsymbol{S}_{1}, \boldsymbol{B}_{\mathrm{NL}} \mathrm{d} V \\
{ }_{r}^{t} \boldsymbol{K}_{\sigma_{2}} & =\int_{r_{V^{(l)}}}{ }_{r} \boldsymbol{B}_{\mathrm{NL}}^{\mathrm{t}}{ }_{r}^{t} \boldsymbol{S}_{2}, \boldsymbol{B}_{\mathrm{NL}} \mathrm{d} V
\end{aligned}
$$

where ' ${ }_{r} S_{1}$ and ' $S_{2}$ are obtained by substituting the 'stresses' $\sigma_{1}$ and $\sigma_{2}$ given by (43b) and (43c) into the expression of matrix ${ }_{r}^{t} S$ of Eq. (35d), respectively (see also Appendix B).

The condition $\left|{ }_{r}^{t_{c}} \boldsymbol{K}_{\mathrm{T}}\right|=0$ yields a quadratic eigenvalue problem which can be solved for the minimum value of $\lambda$, thus giving an approximation of the critical displacement by ${ }^{t_{c}} \boldsymbol{a}={ }^{t} \boldsymbol{a}+\lambda \boldsymbol{\phi}$. Obviously, this process can be simplified by neglecting the quadratic terms in (45). The standard linear eigenvalue problem to be solved now reads simply

$$
\left|{ }_{r}^{i} K_{\mathrm{T}}+\lambda\left({ }_{r}^{t} \boldsymbol{K}_{\mathrm{L}_{2}}+{ }_{r}^{t} \boldsymbol{K}_{\sigma_{1}}\right)\right|=0
$$

The critical load increment can be subsequently estimated from the incremental secant relationship (37) as

$$
\Delta f_{\mathrm{c}}={ }^{t} \boldsymbol{K}_{\mathrm{S}}(\lambda \phi) \lambda \phi=\left[{ }_{r}^{t} \boldsymbol{K}_{\mathrm{T}}\left({ }^{t} a\right)+{ }_{,}^{t} \boldsymbol{K}_{\mathrm{M}}(\lambda \phi)+{ }_{,}^{t} \boldsymbol{K}_{\mathrm{N}}\left(\lambda^{2} \phi^{2}\right)\right] \lambda \phi
$$

where the expression of all matrices coincides with that given in Eqs. (35) and (39).

The estimated critical load vector is finally obtained as

$$
{ }^{t_{\mathrm{c}}} \boldsymbol{f}={ }^{t} \boldsymbol{f}+\Delta \bar{f}_{\mathrm{c}}
$$

where $\Delta \bar{f}_{\mathrm{c}}$ is the projection of $\Delta f_{\mathrm{c}}$ computed from (48) in the direction of the nodal load vector, i.e. after eliminating the spurious contributions associated to nodal load components not included in ${ }^{t} f$.

Obviously, the critical load vector can be computed in a single step from the total secant expression ${ }^{{ }^{c}} \boldsymbol{f}={ }_{{ }_{1}}^{t} \boldsymbol{K}_{\mathrm{S}}\left({ }^{t} \boldsymbol{a}\right){ }^{t_{\mathrm{c}} \boldsymbol{a}}$ (see Fig. 2). However the incremental procedure described above has proved to be more accurate in practice.

\section{Computational strategies}

The approach proposed above can be applied in different ways so as to obtain different approximations to the critical load value.

\subsection{Method I. One step prediction}

(1) Compute the displacement vector ${ }^{0} a$ for a small value of the external forces so that infinitesimal theory still holds.

(2) Take $\phi={ }^{0} a$ as the estimate of the critical displacement increment pattern.

(3) Solve the linear eigenvalue problem (47) for the smallest non-zero eigenvalue.

(4) Estimate the critical load by Eqs. (48) and (49).

This process is comparable in cost to the standard 'initial' stability problem in struts, plates, shells, etc. based in the solution of the eigenvalue problem $[40,41]$

$$
\left|{ }_{r}^{t} \boldsymbol{K}_{\mathbf{L}_{0}}+\lambda{ }_{r}^{t} \boldsymbol{K}_{\sigma}\right|=0
$$

where the smallest non-zero eigenvalue defines the increasing factor of the initial loading ${ }^{0} f$ to give the so-called 'buckling' load as $\lambda^{n} f$. However, it is well known that in many problems this 'initial stability' load can be considerably larger than the actual limit or bifurcation load. The one step 'critical 
displacement' approach proposed here has been proved to give a much more accurate prediction of the critical load as shown in the examples presented in the next section.

\subsection{Method II. Incremental prediction}

(1) Compute the displacement vector ${ }^{t} \boldsymbol{a}$ for each load level ${ }^{t} \boldsymbol{f}$ in the standard incremental manner.

(2) Take $\boldsymbol{\phi}={ }^{t} \boldsymbol{a}$ as the estimate of the critical displacement increment pattern.

(3) and (4) as in Method I.

This approach differs from the previous one in that the critical load is estimated each time that a new displacement configuration ${ }^{i} \boldsymbol{a}$ is found. Naturally a standard stability computation can be also performed at each equilibrium configuration. This implies the solution of the eigenvalue problem

$$
\left|{ }^{t} \boldsymbol{K}_{\mathrm{L}}+\lambda{ }^{t} \boldsymbol{K}_{\sigma}\right|=0
$$

and the stability load is subsequently computed as $\lambda^{\prime} f$.

Obviously, the values of the critical load estimated by the two procedures should converge to the 'exact' value as the solution approaches the instability configuration. The examples analyzed show that the values of the critical load predicted by the critical displacement approach proposed here are in all cases much more accurate than those given by the standard stability method.

\subsection{Method III. Enhanced incremental prediction}

(1) Compute the displacement vector ${ }^{0} a$ corresponding to an initial load level ${ }^{0} f$ in the standard incremental manner (here ${ }^{0} f$ can be taken small cnough so as to give initial displacements within the infinitesimal theory range).

(2) Take $\phi={ }^{0} a$ as the estimate of the critical displacement increment pattern.

(3) and (4) as in Method I.

(5) Compute the stresses, the residual force vector ${ }_{r}^{t_{c}} \boldsymbol{r}$ and the critical load ${ }^{t_{\mathrm{c}}} \boldsymbol{f}$ corresponding to the predicted critical displacement ${ }^{t^{c}} \boldsymbol{a}=(1+\lambda)^{0} a$ using Eqs. (33a), (48) and (49).

The fact that the critical displacement values predicted are close to an equilibrium configuration corresponding to a load level ${ }^{{ }^{c} \mathrm{c}} \boldsymbol{f}$ is now exploited as described next.

(6) Perform an equilibrium iteration to find corrected values of the predicted critical displacement ${ }^{t_{\mathrm{c}} \boldsymbol{a}}$ in equilibrium with the external loads ${ }^{i} f$. For this purpose the standard Newton-Raphson technique can be used as

$$
\begin{aligned}
& \Delta \boldsymbol{a}^{n}=-\left[{ }_{r}^{t_{\mathrm{c}}} \boldsymbol{K}_{\mathrm{T}}^{n}\right]^{-1}{ }_{r}^{t_{c}} \boldsymbol{r}^{n} \\
& { }^{t_{c}} \boldsymbol{a}^{n+1}={ }^{i} \boldsymbol{a}^{n}+\Delta \boldsymbol{a}^{n}
\end{aligned}
$$

with

$$
{ }^{t} \boldsymbol{a}^{0}={ }^{t}{ }^{t} \boldsymbol{a}
$$

(7) Restart the process from Step (2) taking $\phi={ }^{t} \boldsymbol{a}$, where ${ }^{t^{t}} \boldsymbol{a}$ is the converged displacement vector from Step (6).

This method allows very accurate critical loads to be computed in two or three steps as shown in the examples presented next.

\section{Examples. Applications to the analysis of trusses}

The methodology proposed in previous sections will be applied next to the non-linear analysis of truss structures using simple two node linear elements and an updated Lagrangian formulation. The expression of the relevant matrices involved in the computations are given in Appendix C. Further details can be found in [22]. 


\subsection{Two bar truss under central point load}

This well-known example shown in Fig. 5 was analyzed in detail in [22]. The secant stiffness equation relating the vertical displacement of point 2 and the applied load is $[22,27]$.

$$
\left[{ }^{0} k\left({ }^{0} S\right)^{2}+\frac{3}{2}{ }^{0}\left(\frac{k}{l}\right){ }^{0} S v_{2}+\frac{{ }^{0} k}{2\left({ }^{0} l\right)^{2}}\left(v_{2}\right)^{2}\right] v_{2}=-\frac{P}{2}
$$

where ${ }^{0} k={ }^{0}(E A / l),{ }^{0} S=\sin ^{0} \theta$ and ${ }^{0}(\cdot)$ denotes values in the initial (undeformed) configuration.

Let us now estimate the critical load path using the approach proposed in the paper. A general expression of the tangent matrix in terms of the only d.o.f. involved $v_{2}$ can be obtained by simple derivation of Eq. (54), i.e.

$$
{ }_{0}^{t} \boldsymbol{K}_{\mathrm{T}}={ }^{0} \boldsymbol{k}\left[\left({ }^{0} S\right)^{2}+3{ }^{0}\left(\frac{S}{l}\right) v_{2}+\frac{3}{2^{0} l^{2}}\left(v_{2}\right)^{2}\right]
$$
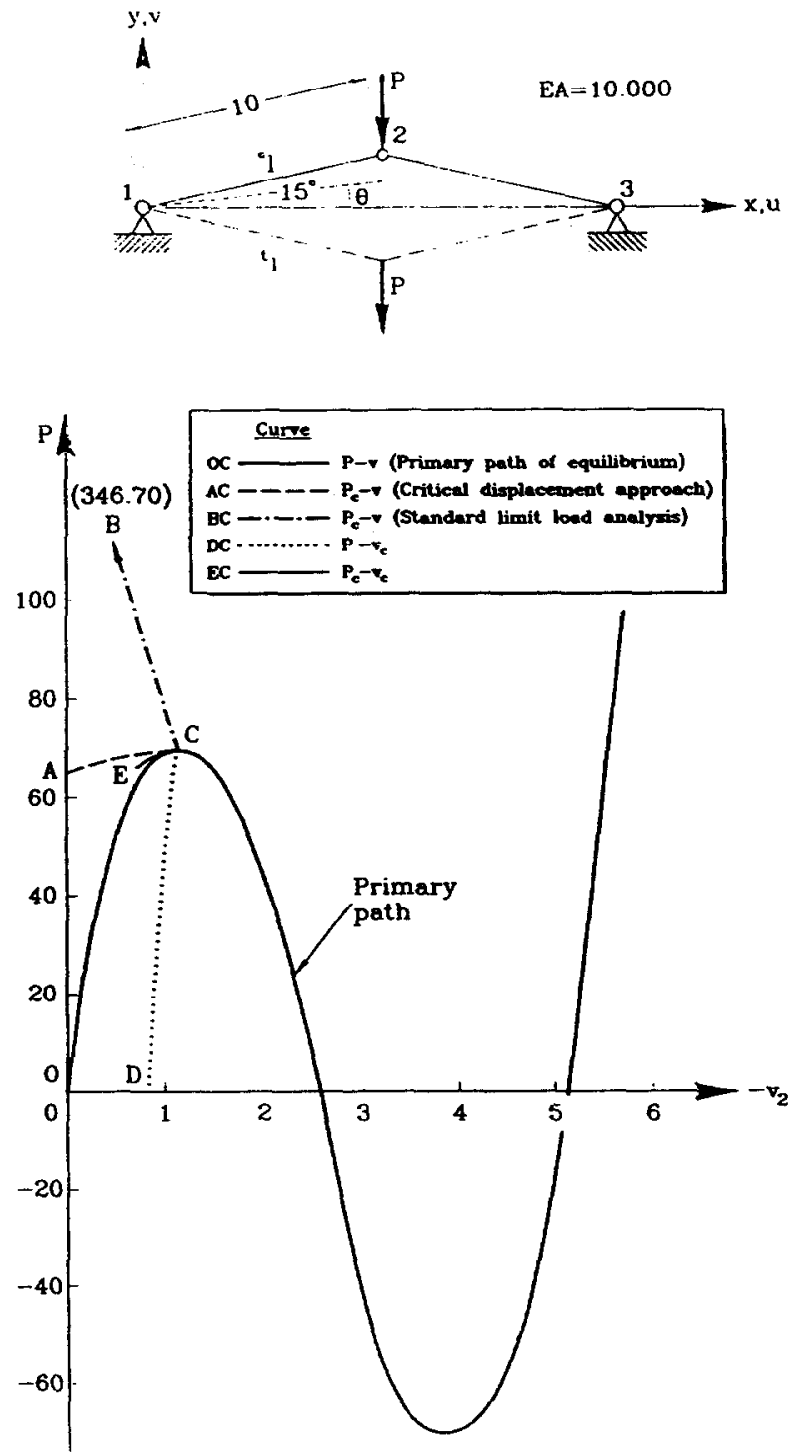

Fig. 5. Two bar truss under point load. Geometry, load-displacement curve and limit load estimates. 
The conditioon ${ }_{0}^{t} K_{\mathrm{T}}=0$ allows the critical displacement to be obtained by solving the corresponding quadratic equation giving

$$
\left(v_{2}\right)_{\mathrm{crit}}={ }^{0} S^{0} l\left[-1 \pm \frac{\sqrt{3}}{3}\right]=\left\{\begin{array}{l}
-1.0939(1.56 \% \text { error }) \\
-4.0825(0.43 \% \text { error })
\end{array}\right.
$$

and the corresponding critical load using Eq. (54) as

$$
P_{\text {crit }}=\frac{ \pm 2^{0} k\left({ }^{0} S\right)^{30} l}{9} \sqrt{3}= \pm 66.732(3.38 \% \text { error })
$$

Above values have been compared with the 'exact' analytical ones taken from [27] giving

$$
\begin{aligned}
& \left(v_{2}\right)_{\text {crit }}^{\text {exact }}= \pm^{0} l \cos ^{0} \theta \tan \left[\operatorname{arcos}\left(\left(\cos { }^{0} \theta\right)^{1 / 3}\right)\right]-{ }^{0} l \sin ^{0} \theta=\left\{\begin{array}{l}
-1.1112 \\
-4.0652
\end{array}\right. \\
& P_{\text {crit }}^{\text {exact }}=2^{0}(E A) \cos ^{0} \theta \tan ^{3}\left[\operatorname{arcos}\left(\left(\cos ^{0} \theta\right)^{1 / 3}\right)\right]= \pm 69.068
\end{aligned}
$$

A 'simpler' estimation of the critical values can be derived by neglecting the quadratic term in (55) yielding

$$
{ }_{0}^{t_{c}} K_{\mathrm{T}} \simeq{ }^{0} k\left[{ }^{0} S^{2}+\frac{3^{0} S}{{ }^{0} l} v_{2}\right]
$$

and the approximate critical displacement and force are now

$$
\begin{aligned}
& \left(v_{2}\right)_{\text {crit }}=\frac{-{ }^{0} l{ }^{0} S}{3}=-0.863(22.36 \% \text { relative error }) \\
& P_{\text {crit }}=\frac{10}{27}{ }^{0} k^{0} l\left({ }^{0} S\right)^{3}=64.20(7.03 \% \text { relative error })
\end{aligned}
$$

These values are quite acceptable given the simplified procedure used and the fact that the prediction has been made from the initial unloaded configuration. Fig. 5 shows the evolution of the critical load obtained using the linearized form and the incremental procedure described in Eqs. (52) and (53). Note the accuracy of the predictions (curve $A C$ ) compared with those obtained using a standard limit load analysis (curve $B C$ ). Further details on this problem can be found in [22].

\section{2. $2 D$ truss beam}

Fig. 6 shows the geometry of this example taken from [42]. The beam, formed by truss elements, is subjected to an increasing horizontal load acting at its left end, as shown in the figure.

The critical load path has been predicted using the method II proposed in this paper. The resulting curve obtained $(A C)$ is plotted in Fig. 5 where the load path predicted using standard limit load analysis is plotted in curve $B C$ in the same figure. Note the accuracy of the predictions based on the critical displacement approach proposed here which gives less than $34 \%$ error in the first critical load value predicted from a simple initial infinitesimal solution. This error is reduced to $0.58 \%$ in only three steps as shown in Table 1.

The primary equilibrium path showing snap-back behaviour has been obtained by combining the standard Newton-Raphson incremental procedure with cylindrical arc-length control [47].

\section{3. $3 D$ pin-jointed star dome structures. Limit load analysis}

The geometry and material properties of the $3 \mathrm{D}$ pin-jointed star dome structure analyzed first is shown in Fig. 7. A vertical point load acting on node 1 is first considered [43].

Fig. 7 displays the limit load paths predicted using the critical displacement approach proposed (curve $A E$ ) and standard limit load analysis (curve $B C$ ). The greater accuracy of the predictions based on the new approach, giving $14.82 \%$ error in the first predicted value bascd on a simple infinitesimal solution, is obvious in this case. This error is reduced to $1.92 \%$ in three steps (see Table 2). 

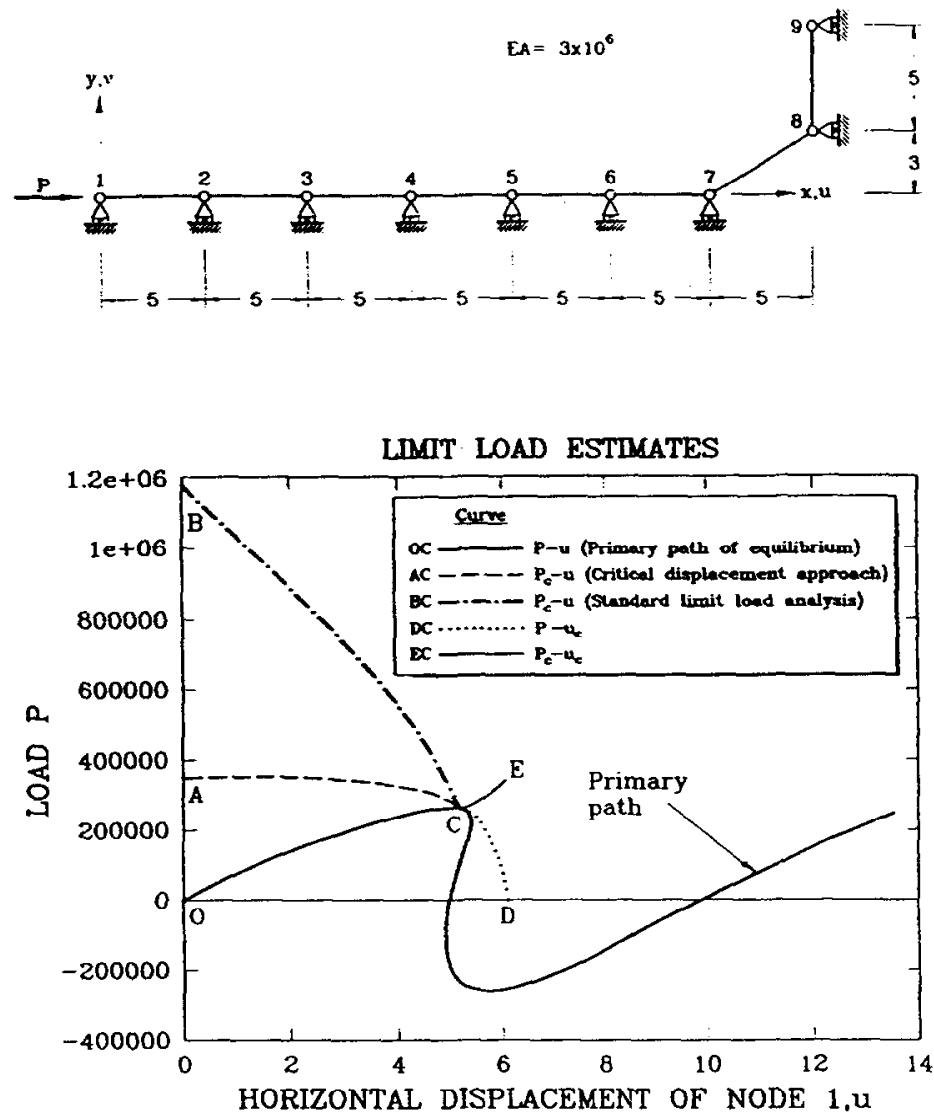

Fig. 6. 2D truss beam. Geometry, load-displacement curve and limit load estimates.

Table 1

2D truss beam

\begin{tabular}{llll}
\hline Load step & \multicolumn{2}{l}{ Critical displacement approach } & \multicolumn{2}{l}{ Limit load analysis } \\
\cline { 2 - 4 } & $\left(u_{\mathrm{C}}\right)_{1}$ & $P_{\mathrm{C}}$ & $P_{\mathrm{C}}$ \\
\hline 1 & $6.05(17.58 \%)$ & $3.52 \times 10^{5}(34.24 \%)$ & $9.85 \times 10^{5}(275.26 \%)$ \\
2 & $5.83(13.27 \%)$ & $3.23 \times 10^{5}(22.96 \%)$ & $5.88 \times 10^{5}(124.03 \%)$ \\
3 & $5.00(2.84 \%)$ & $2.617 \times 10^{5}(0.34 \%)$ & $3.52 \times 10^{5}(34.00 \%)$ \\
\hline
\end{tabular}

Critical displacement $u_{\mathrm{C}}$ of node 1 and critical load $P_{\mathrm{C}}$ were obtained using the critical displacement approach (Method III) and standard limit load analysis (Eq. (51))

Numbers in brackets show percentage error with respect to the 'exact' solution: $u_{\mathrm{C}}=5.146$ and $P_{\mathrm{C}}=2.626 \times 10^{-3}[42]$.

Fig. 8 shows results for an analysis performed for the same structure with vertical loads acting now in nodes 1 to 7 [44]. The limit load paths predicted are also displayed in Fig. 8 showing again the superiority of the critical displacement approach (see also Table 3).

A similar example is shown in Fig. 9 where details of the geometry, loading and material properties is given. Again the limit load path predicted with the critical displacement method gives remarkable accurate values even for the first prediction based on an infinitesimal solution (see Table 4).

\section{4. $3 D$ pin-jointed truss dome. Prediction of bifurcation load}

This example shows the ability of the approach to predict the bifurcation load in a 3D truss structure [19]. The geometry, loading and material properties of the structure are displayed in Fig. 10. The critical load paths obtained with the critical displacement procedure (curve $A C$ ) and standard limit load analysis (curve $B C$ ) are shown in Fig. 10. A bifurcation point is detected by both procedures for 

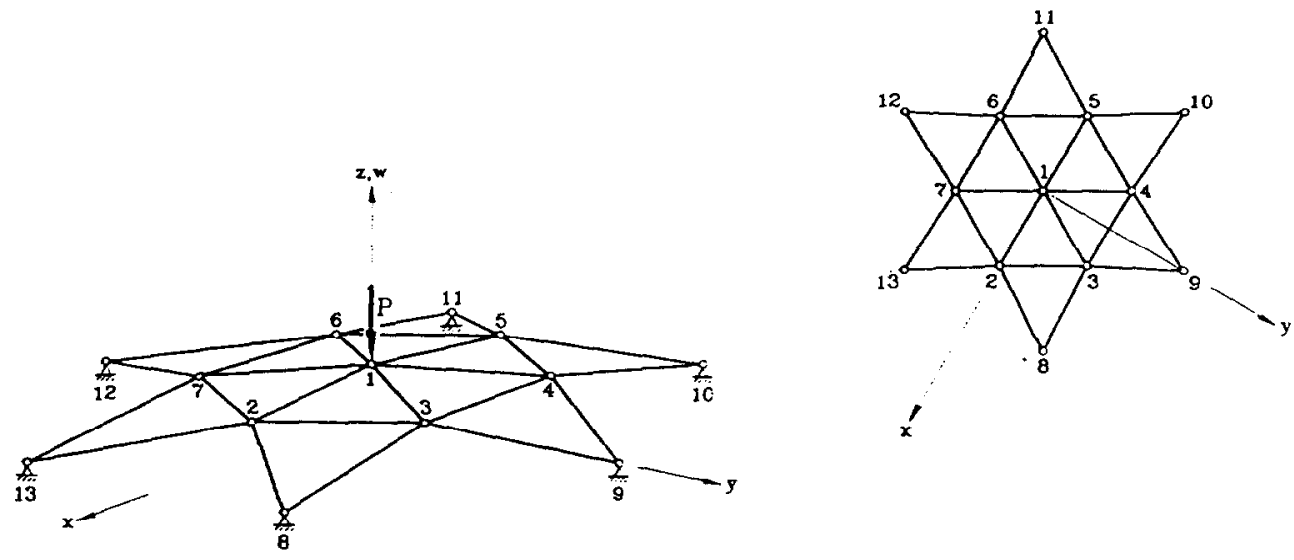

$$
x^{\prime}
$$

$\mathrm{EA}=10^{4}$
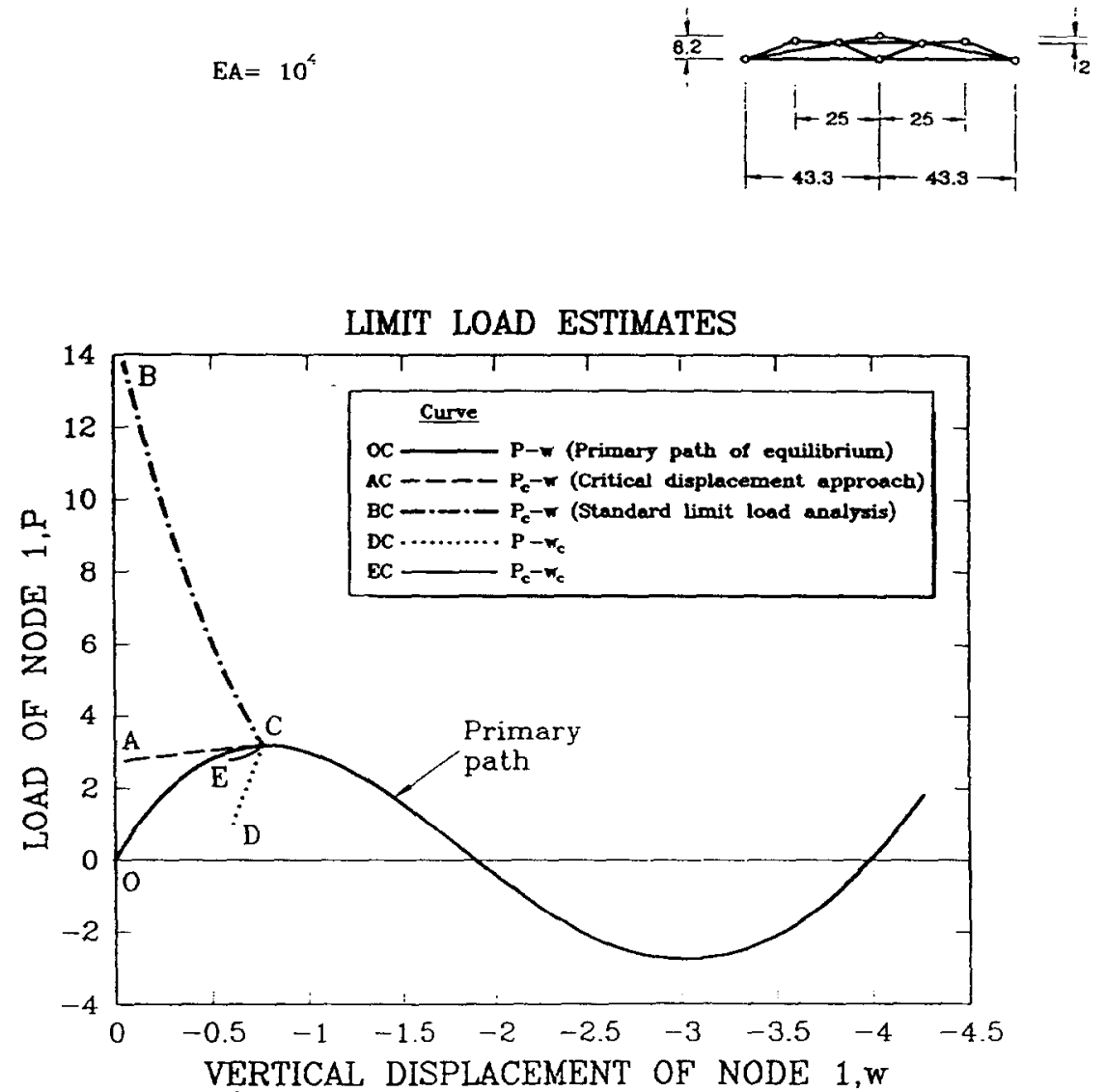

Fig. 7. 3D pin-jointed star under central point load. Geometry, load-displacement curve and limit load estimates.

Table 2

13 node star truss dome under central point load

\begin{tabular}{llll}
\hline Load step & \multicolumn{2}{l}{ Critical displacement approach } & \multicolumn{2}{l}{ Limit load analysis } \\
\cline { 2 - 4 } & $\left(w_{\mathrm{C}}\right)_{\mathrm{t}}$ & $P_{\mathrm{C}}$ & $P_{\mathrm{C}}$ \\
\hline 1 & $-0.405(28.25 \%)$ & $69.46(12.94 \%)$ & $365.37(357.95 \%)$ \\
2 & $-0.540(4.42 \%)$ & $77.02(3.46 \%)$ & $135.74(70.14 \%)$ \\
3 & $-0.556(1.51 \%)$ & $79.02(0.95 \%)$ & $91.37(14.52 \%)$ \\
\hline
\end{tabular}

Critical displacement $\left(w_{c}\right)$ of node 1 and critical load were obtained using the critical displacement approach (Method III) and standard limit load analysis (Eq. (51)).

Numbers in brackets show percentage error with respect to the 'exact' solution: $w_{\mathrm{C}}=-0.516$ and $P_{\mathrm{C}}=79.50[43]$ 

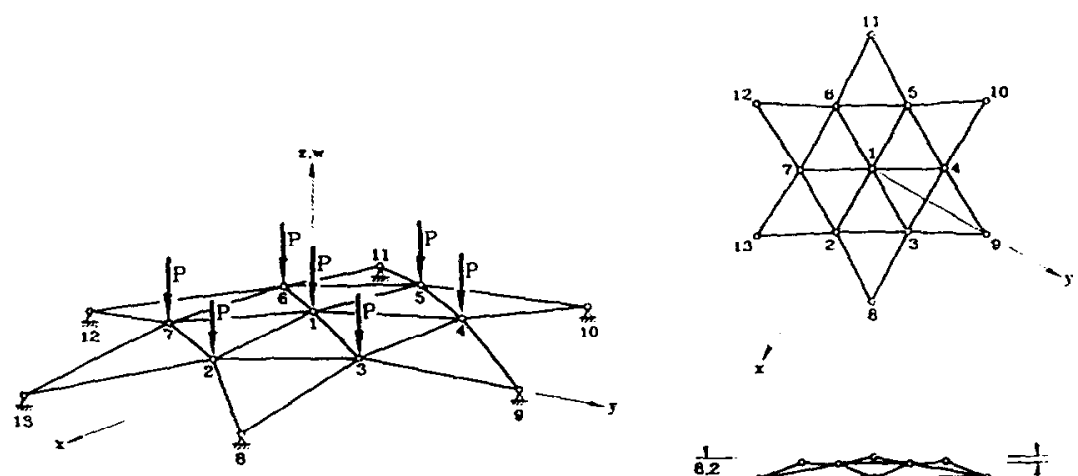

$E A=10^{4}$
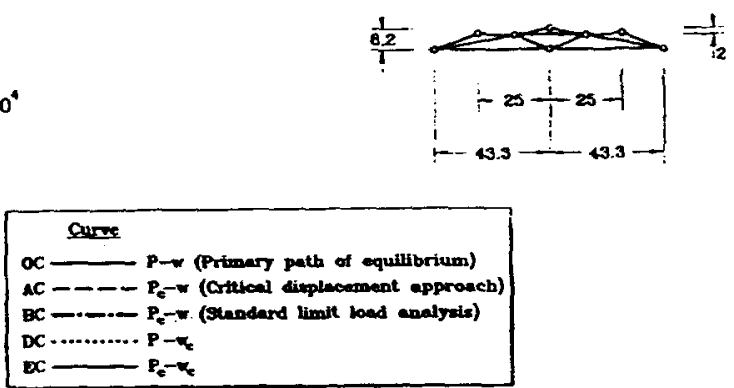

LMIT LOAD ESTMATES

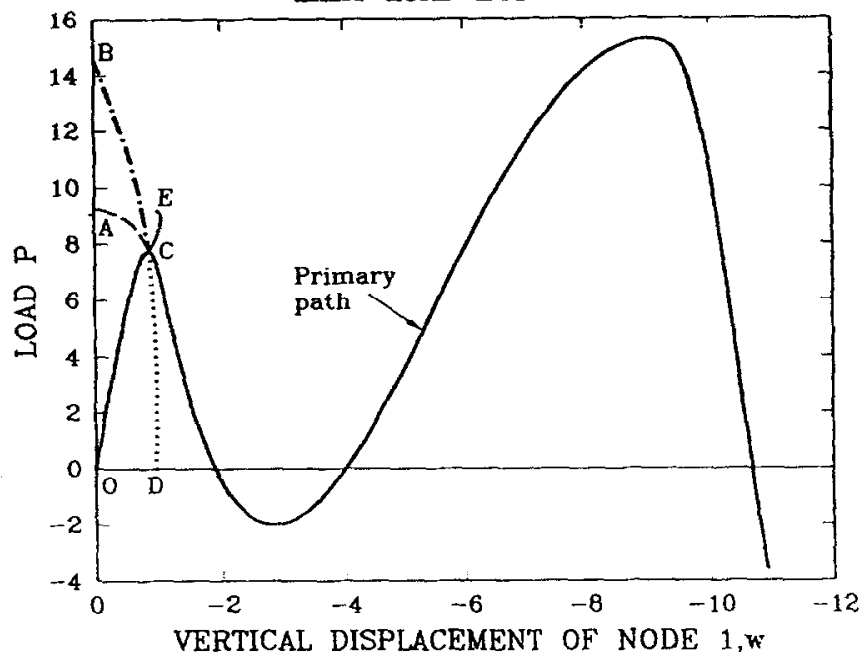

Fig. 8. 3D pin-jointed star under seven point loads. Geometry, load-displacement curve and limit load estimates.

Table 3

13 node star truss dome under seven point loads

\begin{tabular}{llll}
\hline Load step & \multicolumn{2}{l}{ Critical displacement approach } & \multicolumn{2}{l}{ Limit load analysis } \\
\cline { 2 - 4 } & $\left(w_{\mathrm{C}}\right)_{1}$ & $P_{\mathrm{C}}$ & $P_{\mathrm{c}}$ \\
\hline 1 & $-0.876(0.69 \%)$ & $9.22(18.86 \%)$ & $14.53(87.20 \%)$ \\
2 & $-0.896(1.66 \%)$ & $8.42(8.56 \%)$ & $9.88(27.40 \%)$ \\
3 & $-0.887(0.61 \%)$ & $7.97(2.67 \%)$ & $8.01(3.23 \%)$ \\
\hline
\end{tabular}

Critical displacement $w_{c}$ of node 1 and critical load were obtained using the critical displacement approach (Method III) and standard limit load analysis (Ey. (51)).

Numbers in brackets show percentage error with respect to the 'exact' solution: $w_{\mathrm{C}}=-0.882$ and $P_{\mathrm{C}}=7.81$ [44]. 


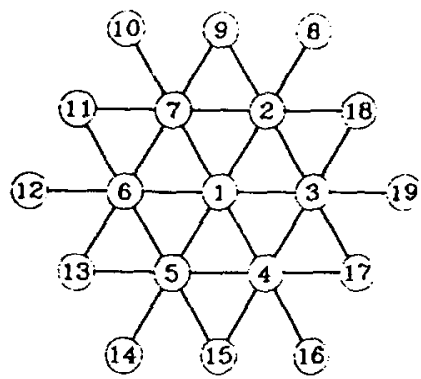

Vertical load $P$ acting on node

$E A=10^{\circ}$

\begin{tabular}{|cccc|}
\hline \multicolumn{4}{c}{ COORDDATSS OF THE NODE PONTS OF DOIE STRUCTURE } \\
\hline Node & $X$ & $Y$ & $Z$ \\
\hline 1 & 0.0 & 0.0 & 6.0 \\
3 & -15.0 & 25.9807 & 4.5 \\
$4-$ & -30.0 & 0.0 & 4.5 \\
9 & 0.0 & 60.0 & 0.0 \\
10 & -30.0 & 51.9615 & 0.0 \\
11 & -54.9615 & 30.0 & 0.0 \\
12 & -60.0 & 0.0 & 0.0 \\
\hline
\end{tabular}

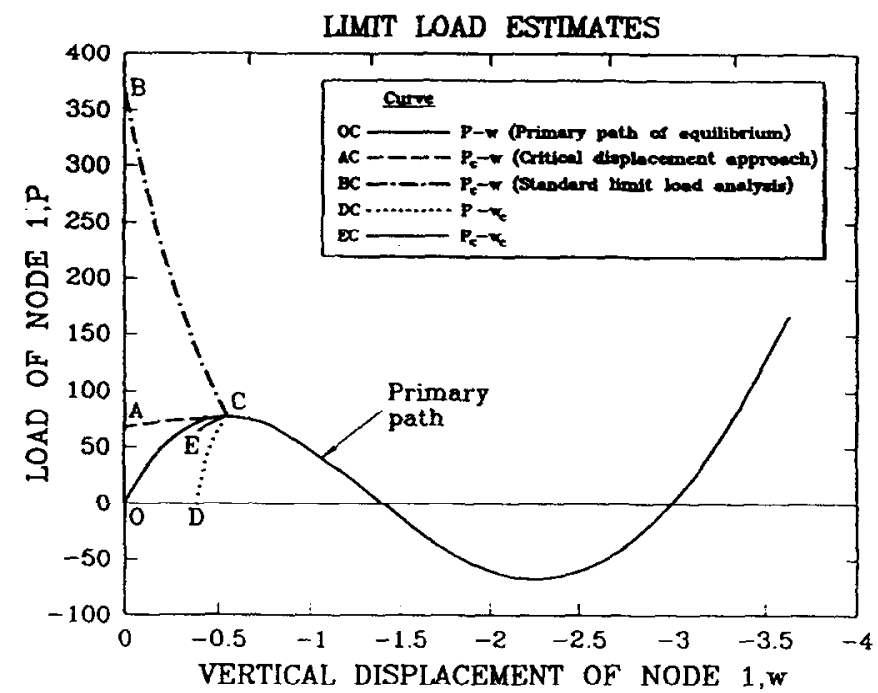

Fig. 9. 19 node star truss dome under central point load. Geometry, load-displacement curve and limit load estimates.

Table 4

19 node star truss dome under central point loads

\begin{tabular}{llll}
\hline Load step & \multicolumn{2}{l}{ Critical displacement approach } & \multicolumn{2}{l}{ Limit load analysis } \\
\cline { 2 - 3 } & $\left(w_{\mathrm{C}}\right)_{1}$ & $P_{\mathrm{C}}$ & $P_{\mathrm{C}}$ \\
\hline 1 & $-0.571(25.02 \%)$ & $2.69(14.82 \%)$ & $14.54(360.72 \%)$ \\
2 & $-0.750(1.48 \%)$ & $3.02(4.26 \%)$ & $5.43(72.24 \%)$ \\
3 & $-0.769(1.02 \%)$ & $3.096(1.92 \%)$ & $3.98(25.99 \%)$ \\
\hline
\end{tabular}

Critical displacement $w_{C}$ of node 1 and critical load were obtained using the critical displacement approach (Method III) and standard limit load analysis (Eq. (51)).

Numbers in brackets show percentage error with respect to the 'exact' solution: $w_{\mathrm{C}}=-0.761$ and $P_{\mathrm{C}}=3.157$ [45]. 
(a)
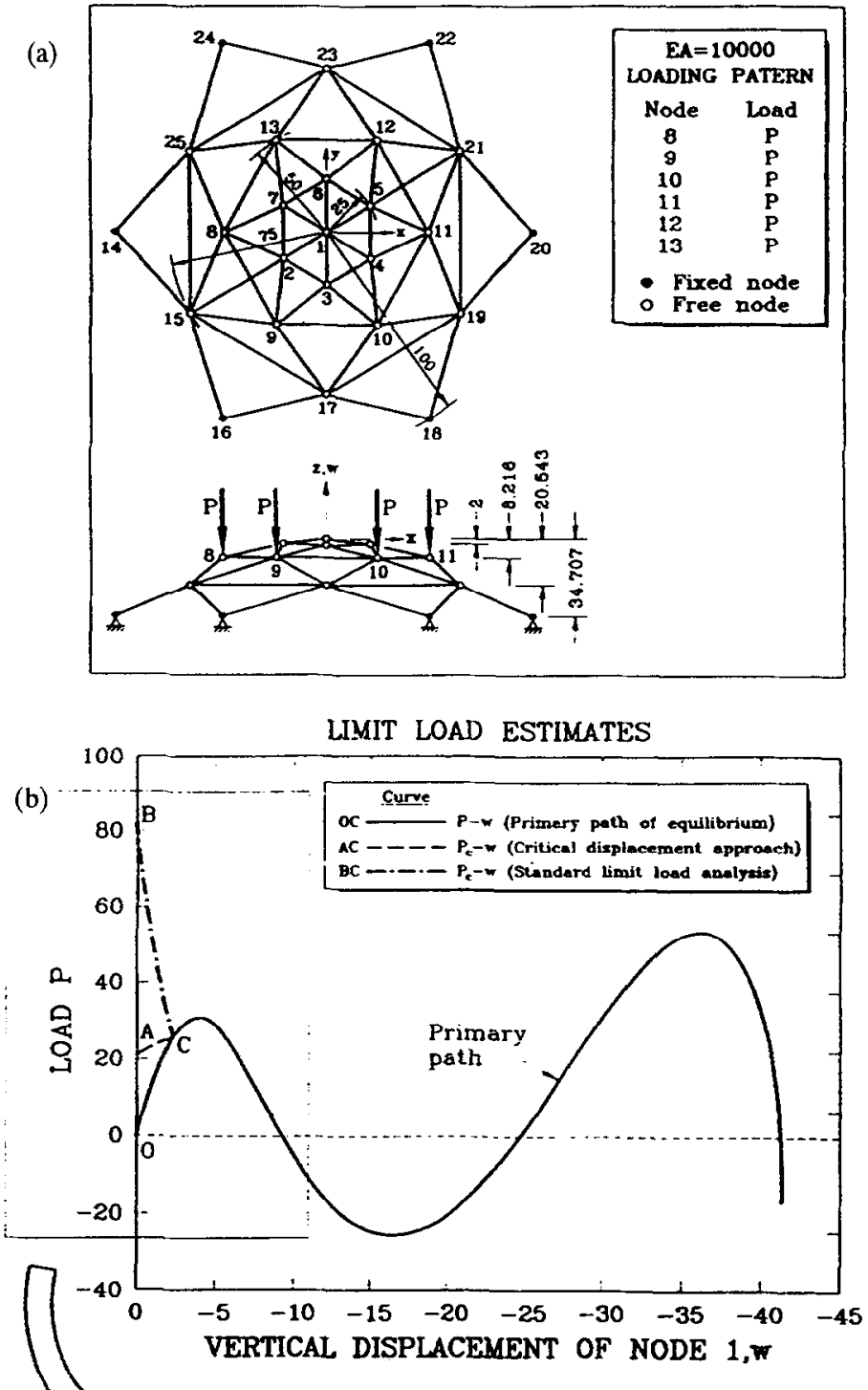

LMTT LOAD ESTMATES

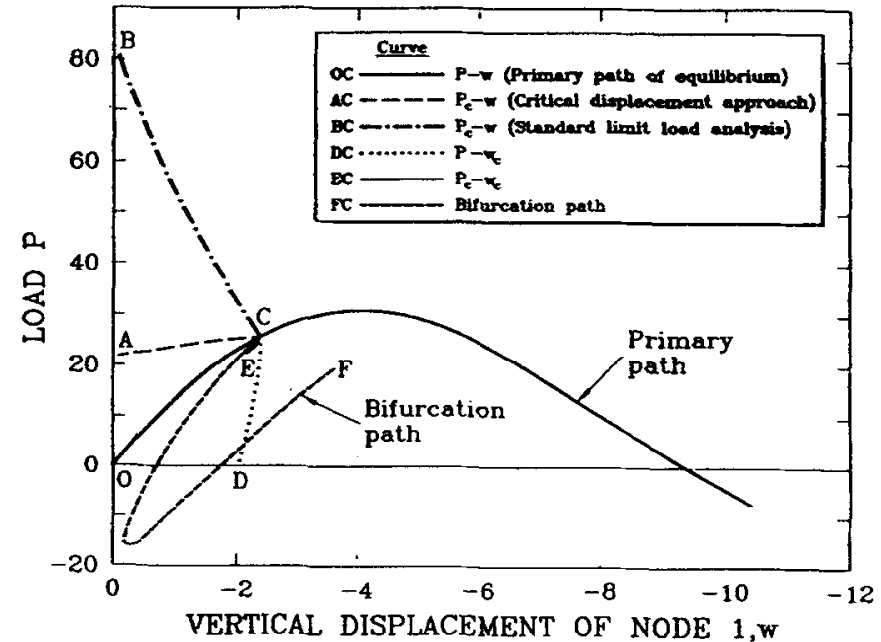

Fig. 10. 25 node star truss dome. (a) Definition of geometry and loading; (b) load-displacement curve and limit load estimates. 
Table 5

25 node star truss dome under four point loads

\begin{tabular}{llll}
\hline Load step & \multicolumn{2}{l}{ Critical displacement approach } & \multicolumn{2}{l}{ Limit load analysis } \\
\cline { 2 - 3 } & $\left(w_{\mathrm{C}}\right)_{1}$ & $P_{\mathrm{C}}$ & $P_{\mathrm{C}}$ \\
\hline 1 & $-2.05(18.23 \%)$ & $21.71(19.21 \%)$ & $79.79(196.90 \%)$ \\
2 & $-2.37(5.44 \%)$ & $25.39(5.72 \%)$ & $33.35(24.10 \%)$ \\
3 & $-2.42(3.80 \%)$ & $26.69(2.16 \%)$ & $24.92(7.27 \%)$ \\
\hline
\end{tabular}

Critical (bifurcation) load was obtained using the critical displacement approach (Method III) and standard limit load analysis (Eq. (5)).

Numbers in brackets show percentage error with respect to the 'exact' solution: $w_{\mathrm{C}}=-2.516$ and $P_{\mathrm{C}}=26.89[19]$.

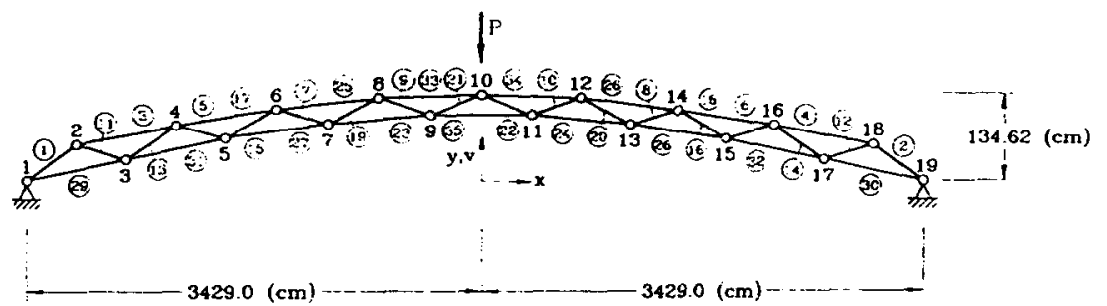

\begin{tabular}{|c|c|c|c|c|}
\hline \multicolumn{3}{|c|}{ NODAL COORDINATES } & \multicolumn{2}{|c|}{ CROSS SECTIONAL AREAS } \\
\hline Nodal number & z Coordinate & $\times$ Coordinate & Ber's number & Area $\left(\mathrm{cm}^{2}\right)$ \\
\hline 1. 19 & $\mp 3429.0$ & 0.00 & $1-10.35$ & 51.61 \\
\hline 2. 18 & 73048.0 & 50.65 & 11.12 & 64.52 \\
\hline 3. 17 & $\mp 2667.0$ & 34.75 & $13-16$ & 83.87 \\
\hline 4. 16 & $\mp 2286.0$ & 63.82 & 17. 18 & 96.77 \\
\hline 5.15 & 71905.0 & 65.30 & $19-22$ & 103.23 \\
\hline 6. 14 & $\mp 1524.0$ & 110.85 & 23.24 & 161.29 \\
\hline 7. 13 & $\mp 1143.0$ & 87.99 & 25.26 & 195.55 \\
\hline 6. 12 & $\mp 762.0$ & 128.50 & 27. 28 & 258.06 \\
\hline 9. 11 & 7381.0 & 100.05 & $29-32$ & 290.32 \\
\hline 10 & 0.0 & 134.60 & 33,34 & 308.68 \\
\hline
\end{tabular}

Young's modulus is $7.03 \times 10^{5}\left(\mathrm{kgf} / \mathrm{cm}^{2}\right)$

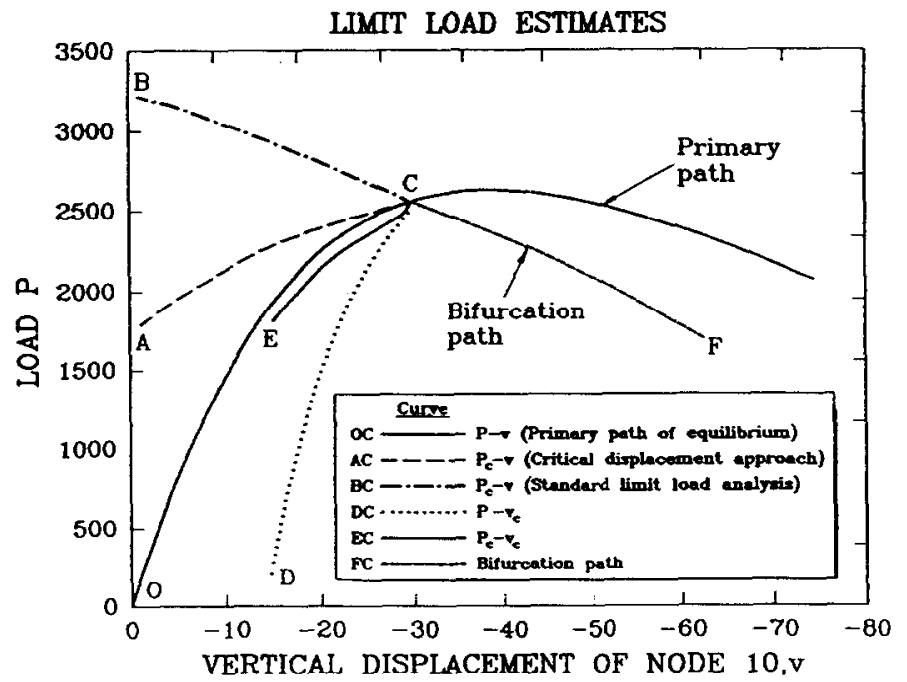

Fig. 11. Arch-truss structure under central point load. Geometry, load-displacement curve and limit load estimates. 
Table 6

Arch-truss structure

\begin{tabular}{llll}
\hline Load step & \multicolumn{2}{l}{ Critical displacement approach } & \multicolumn{1}{l}{ Limit load analysis } \\
\cline { 2 - 3 } & $\left(v_{\mathrm{C}}\right)_{\mathrm{C}}$ & $P_{\mathrm{C}}$ & $P_{\mathrm{C}}$ \\
\hline 1 & $-14.70(52.09 \%)$ & $1795.4(30.40 \%)$ & $3223.0(24.99 \%)$ \\
2 & $-23.42(23.73 \%)$ & $2308.0(10.53 \%)$ & $2917.6(13.10 \%)$ \\
3 & $-29.51(3.87 \%)$ & $2518.9(2.36 \%)$ & $2632.9(2.06 \%)$ \\
\hline
\end{tabular}

Critical (bifurcation) load was obtained using the critical displacement approach (Method III) and standard limit load analysis (Eq. (51))

Numbers in brackets show percentage error with respect to the 'exact' solution: $v_{\mathrm{C}}=-30.70$ and $P_{\mathrm{C}}=2578.41[46]$.

$(P=26.89)$. Notc the higher accuracy of the prediction based in the critical displacement procedure. The first estimate of the critical load based on the simple infinitesimal solution gives a $19.21 \%$ error. This error is reduced to $2.16 \%$ in only three steps (see Table 5). The antisymmetric bifurcation path is plotted in curve $C D$ of Fig. 10. This has been obtained by perturbing the geometry of the structure at the critical load using the first eigenmode for this load level and then using an arc length technique.

\subsection{Arch-truss structure. Prediction of bifurcation load}

The geometry of the arch is described in Fig. 11 together with details of the mechanical properties [46]. A vertical point load acts on the central node as shown in the figure.

Fig. 11 shows the critical load paths predicted with the critical displacement approach proposed (curve $A C$ ) and the standard stability analysis (curve $B C$ ). A bifurcation point is detected in this case for $P=2578.41$. Note that this critical load value is estimated with $2.36 \%$ error in only 3 steps (see Table 6).

\section{Conclusions}

The critical displacement (CD) approach proposed seems to be a simple and effective procedure for computing structural instability points. The cost of the computation is comparable to that of standard stability analysis and the accuracy has proved to be superior in all cases studied. The application of the $\mathrm{CD}$ method for analysis of instability of trusses or any other structures using solid elements is straightforward and all the necessary expressions have been given here. The extension of the CD approach to structural problcms involving rotational degrees of freedom (i.e. beams and shells) requires further study as the existence of the secant stiffness matrix is questionable in this case.

\section{Acknowledgments}

Willian T. Matias gratefully acknowledges the financial support received from the CAPES programme of Brasil.

\section{Appendix A}

The first- and second-order strain increments can be written as

$$
\begin{aligned}
& { }_{r} \boldsymbol{e}=\left[L_{0}+{ }_{r}{ }_{r} L_{\mathrm{l}}\left({ }_{r} g\right)\right]_{r} g \\
& { }_{r} \boldsymbol{\eta}=\frac{1}{2}{ }_{r} L_{1}\left({ }_{r} g\right){ }_{r} g
\end{aligned}
$$




$$
\begin{aligned}
& { }_{r} \boldsymbol{e}=\left[{ }_{r} e_{11},{ }_{r} e_{22},{ }_{r} e_{33}, 2{ }_{r} e_{12}, 2{ }_{r} e_{13}, 2{ }_{r} e_{23}\right]^{\mathrm{T}} \\
& { }_{r} \boldsymbol{\eta}=\left[{ }_{r} \eta_{11},{ }_{r} \eta_{22},{ }_{r} \eta_{33}, 2{ }_{r} \eta_{12}, 2{ }_{r} \eta_{13}, 2{ }_{r} \eta_{23}\right]^{\mathrm{T}}
\end{aligned}
$$

For 3D solids

$$
\begin{aligned}
\boldsymbol{L}_{0} & =\left[\begin{array}{ccc}
100 & 000 & 000 \\
000 & 010 & 000 \\
000 & 000 & 001 \\
010 & 100 & 000 \\
001 & 000 & 100 \\
000 & 001 & 001
\end{array}\right] \\
{ }_{r}^{t} \boldsymbol{L}_{1} & =\left[\begin{array}{cc}
{ }_{r}^{t} \boldsymbol{g}^{\mathbf{T}} & \boldsymbol{H}_{1} \\
{ }^{\prime} \boldsymbol{g}^{\mathbf{T}} & \boldsymbol{H}_{2} \\
& \vdots \\
{ }_{r} \boldsymbol{g}^{\mathbf{T}} & \boldsymbol{H}_{6}
\end{array}\right] ; \quad \boldsymbol{L}_{1}=\left[\begin{array}{cc}
{ }_{r} \boldsymbol{g}^{\mathrm{T}} & \boldsymbol{H}_{1} \\
{ }_{r} \boldsymbol{g}^{\mathrm{T}} & \boldsymbol{H}_{2} \\
& \vdots \\
{ }_{r} \boldsymbol{g}^{\mathrm{T}} & \boldsymbol{H}_{6}
\end{array}\right]
\end{aligned}
$$

where

$$
{ }_{r}^{t} \boldsymbol{g}=\left\{\begin{array}{l}
{ }_{r} g_{1} \\
r \\
t g_{2} \\
r \\
{ }_{r} g_{3}
\end{array}\right\} ; \quad, \boldsymbol{g}=\left\{\begin{array}{l}
{ }_{r} g_{1} \\
r g_{2} \\
r g_{3}
\end{array}\right\}
$$

with

$$
{ }_{r}^{t} g_{i}=\frac{\partial^{t} u}{\partial^{r} x_{i}} ; \quad{ }_{r} g_{i}=\frac{\partial(\Delta u)}{\partial^{r} x_{i}}
$$

and

$$
\begin{array}{lll}
\boldsymbol{H}_{1}=\left[\begin{array}{lll}
\boldsymbol{I}_{3} & \mathbf{0} & \mathbf{0} \\
\mathbf{0} & \mathbf{0} & \mathbf{0} \\
\mathbf{0} & \mathbf{0} & \mathbf{0}
\end{array}\right] ; & \boldsymbol{H}_{2}=\left[\begin{array}{ccc}
\mathbf{0} & \mathbf{0} & \mathbf{0} \\
\mathbf{0} & \boldsymbol{I}_{3} & \mathbf{0} \\
\mathbf{0} & \mathbf{0} & \mathbf{0}
\end{array}\right] \\
\boldsymbol{H}_{3}=\left[\begin{array}{ccc}
\mathbf{0} & \mathbf{0} & \mathbf{0} \\
\mathbf{0} & \mathbf{0} & \mathbf{0} \\
\mathbf{0} & \mathbf{0} & \boldsymbol{I}_{3}
\end{array}\right] ; & \boldsymbol{H}_{4}=\left[\begin{array}{ccc}
\mathbf{0} & \boldsymbol{I}_{3} & \mathbf{0} \\
\boldsymbol{I}_{3} & \mathbf{0} & \mathbf{0} \\
\mathbf{0} & \mathbf{0} & \mathbf{0}
\end{array}\right] \\
\boldsymbol{H}_{5}=\left[\begin{array}{lll}
\mathbf{0} & \mathbf{0} & \boldsymbol{I}_{3} \\
\mathbf{0} & \mathbf{0} & \mathbf{0} \\
\boldsymbol{I}_{3} & \mathbf{0} & \mathbf{0}
\end{array}\right] ; & \boldsymbol{H}_{6}=\left[\begin{array}{lll}
\mathbf{0} & \mathbf{0} & \mathbf{0} \\
\mathbf{0} & \mathbf{0} & \boldsymbol{I}_{3} \\
\mathbf{0} & \boldsymbol{I}_{3} & \mathbf{0}
\end{array}\right] \\
\boldsymbol{I}_{3}=\left[\begin{array}{lll}
1 & 0 & 0 \\
\mathbf{0} & 1 & 0 \\
0 & 0 & 1
\end{array}\right] ; & \mathbf{0}=\left[\begin{array}{lll}
0 & 0 & 0 \\
0 & 0 & 0 \\
0 & 0 & 0
\end{array}\right]
\end{array}
$$

From (A.1) and (A.2)

$$
\begin{aligned}
& \delta_{r} \boldsymbol{e}=\left(\boldsymbol{L}_{0}+{ }_{r}^{t} \boldsymbol{L}_{1}\right) \delta_{r} \boldsymbol{g} \\
& \delta_{r} \boldsymbol{\eta}={ }_{r} \boldsymbol{L}_{1} \boldsymbol{\delta}_{r} \boldsymbol{g}
\end{aligned}
$$


Substituting the finite element interpolation (26) into the expression of ${ }_{r} g$ of (A.5) gives

$$
{ }_{r} g={ }_{r} G \Delta a
$$

where a typical nodal component of the gradient matrix for 3D solids is given by

$$
\boldsymbol{G}^{k}=\left[\begin{array}{ll}
\frac{\partial N^{k}}{\partial^{r} x_{1}} & I_{3} \\
\frac{\partial N^{k}}{\partial^{r} x_{2}} & I_{3} \\
\frac{\partial N^{k}}{\partial^{r} x_{3}} & I_{3}
\end{array}\right]
$$

Combining (A.1), (A.2) and (A.10) gives

$$
\begin{aligned}
& { }_{r} \boldsymbol{e}=\left[L_{0}+{ }_{r}^{t} L_{1}\right], G \Delta a=\left[{ }_{r} B_{\mathrm{L}_{0}}+{ }_{,} B_{\mathrm{L}_{1}}\left({ }^{\prime} a\right)\right] \Delta a \\
& { }_{r} \boldsymbol{\eta}=\frac{1}{2}, L_{1 r} G \Delta a=\frac{1}{2}, B_{1}(\Delta a) \Delta a
\end{aligned}
$$

Where the different nodal components of the first- and second-order strain matrices are obtained by

$$
\begin{aligned}
& { }_{r} B_{\mathrm{L}_{0}}^{k}=L_{0}, G^{k} \\
& { }_{r} B_{\mathrm{L}_{1}}^{k}\left({ }^{i} a\right)={ }_{r} L_{1}\left({ }^{t} a\right), G^{k} \\
& { }_{r} B_{1}(\Delta a)={ }_{r} L_{1}(\Delta a), G^{k}
\end{aligned}
$$

Further details can be found in [22].

\section{Appendix B}

Box B.1

Matrices ${ }^{t} \boldsymbol{E}, \boldsymbol{B}_{\mathrm{NL}},{ }_{r} \boldsymbol{S}$ and ${ }_{r}^{i} \boldsymbol{H}$ for $3 \mathrm{D}$ elastic solids

$$
\begin{aligned}
& { }_{r}^{\prime} \boldsymbol{E}-\left[\begin{array}{ccc}
a I_{3} & d I_{3} & e I_{3} \\
& b I_{3} & f I_{3} \\
s y m . & & c I_{3}
\end{array}\right] ; \quad[a, b, c, d, e, f]^{\top}-{ }_{,}^{\prime} D, e
\end{aligned}
$$

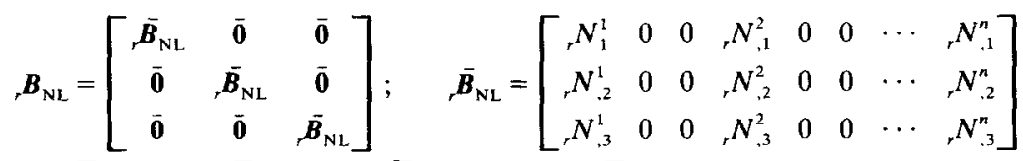

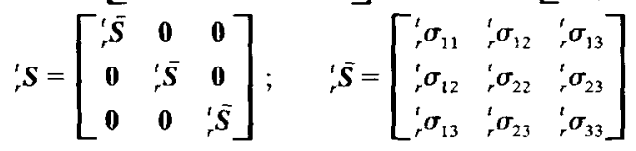

$$
\begin{aligned}
& \mathbf{0}=\left[\begin{array}{ccc}
0 & 0 & 0 \\
0 & 0 & 0 \\
0 & 0 & 0
\end{array}\right] ; \quad \overline{\mathbf{0}}=\left\{\begin{array}{l}
0 \\
0 \\
0
\end{array}\right\} ; \quad{ }_{r} N_{. i}^{k}=\frac{\partial N^{k}}{\partial^{r} x_{i}} \\
& { }^{\prime} \boldsymbol{H}=\sum_{i=1}^{6} \sum_{j=1}^{6}{ }_{r} d_{i j} \eta_{i} \boldsymbol{H}_{i} ; \quad{ }_{r} \boldsymbol{\eta}_{i}={ }_{r} \boldsymbol{g}^{\mathrm{T}} \boldsymbol{H}_{i r} \boldsymbol{g} \\
& { }_{i} d_{i j} \text { : element } i j \text { of constitutive matrix ' } D
\end{aligned}
$$




\section{Appendix C}

\section{C.1. Relevant expression for $3 D$ trusses}

Fig. C.1 displays a typical two node truss element defined in a three-dimensional frame with global and local axes denoted by $x_{i}$ and $x_{i}^{\prime}(i=1.3)$, respectively.

For the sake of precision an updated Lagrangian formulation will be used $\left({ }^{r} V={ }^{t} V\right)$. The first- and second-order axial strain increments are defined as

$$
e_{11}^{\prime}=\frac{\mathrm{d}\left(\Delta u_{1}^{\prime}\right)}{\mathrm{d}^{\prime} x_{1}^{\prime}} ; \quad \eta_{11}^{\prime}=\frac{1}{2} \frac{\mathrm{d}\left(\Delta u_{1}^{\prime}\right)}{\mathrm{d}^{\prime} x_{1}^{\prime}} \frac{\mathrm{d}\left(\Delta u_{1}^{\prime}\right)}{\mathrm{d}^{\prime} x_{1}^{\prime}}
$$

where $\Delta u_{1}^{\prime}$ is the displacement increment along the local axis ' $x_{1}^{\prime}$.

The constitutive equation is simply

$$
{ }_{t} \Delta N={ }^{\prime}[E A]\left[{ }_{t} e_{11}^{\prime}+{ }_{t} \eta_{11}^{\prime}\right]
$$

where,$\Delta N$ is the axial force increment and $E$ and $A$ are, respectively, the Young's modulus and the area of the transverse cross section.

Local and global displacements are related by the standard transformation

$$
\Delta u_{j}^{\prime}=\frac{\partial^{t} x_{i}}{\partial^{t} x_{j}^{\prime}} \Delta u_{i}, \quad i, j=1,2,3
$$

The global displacements are interpolated in the usual manner

$$
\Delta u_{i}=\sum_{k=1}^{2} N^{k}(\xi) \Delta u_{i}^{k}
$$

where $N^{k}(\xi)=\frac{1}{2}\left(1+\xi \xi_{k}\right)$ are the linear shape functions of the standard two node element [41].

The expressions of the relevant matrices required for the computation of the secant required for the computation of the secant stiffness matrix are given in Box C.1. The particular explicit symmetric form of this matrix for $\alpha=1 / 2$ and $\beta=0$ and a truss of constant cross section and homogeneous material is shown in Box C.2.

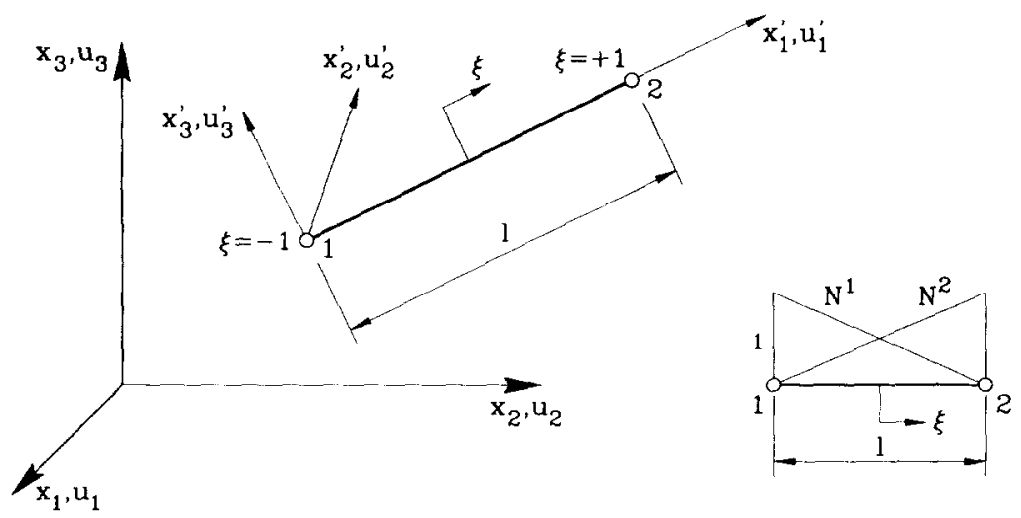

Fig. C.1. 3D two node truss element. 
Box C.1

Relevant expressions involved in the computation of the secant stiffness matrix for linear 3D truss elements

\begin{tabular}{|c|}
\hline 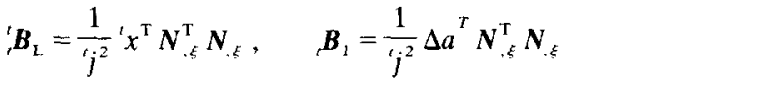 \\
\hline $\boldsymbol{B}_{\mathrm{NL}}={ }_{t} \boldsymbol{G}=\frac{1}{t_{j}} \boldsymbol{N}_{\cdot \xi}, \quad{ }_{t}^{t} \boldsymbol{E}=\frac{{ }^{t}[E A]}{{ }_{j}^{t} j^{2}} \boldsymbol{x}^{\mathrm{T}} \boldsymbol{N}_{\cdot \xi}^{\mathrm{T}} \boldsymbol{N}_{\cdot \xi} \Delta \boldsymbol{a}$ \\
\hline$N_{, \xi}=\left[\frac{\mathrm{d} N^{t}}{\mathrm{~d} \xi} I_{3}, \frac{\mathrm{d} N^{2}}{\mathrm{~d} \xi} I_{3}\right] ; \quad ' j=\frac{\mathrm{d}^{\prime} x_{1}^{\prime}}{\mathrm{d} \xi}\left(\right.$ usually $\left.j=\frac{l^{(e)}}{2}\right)$ \\
\hline$H=\Delta a^{\top}{ }_{r}{ }_{r}{ }^{\mathrm{T}}{ }^{t}[E A]{ }_{r} \boldsymbol{G} \Delta \boldsymbol{a}$ \\
\hline${ }^{\prime} \mathrm{x}=\left[{ }^{\mathrm{t}} x_{1}^{1},{ }^{1}{ }^{t} x_{2}^{1},{ }^{\prime} x_{3}^{1},{ }^{\prime} x_{1}^{2},{ }^{\prime} x_{2}^{2},{ }^{\prime} x_{3}^{2}\right]^{\mathrm{T}}$ \\
\hline$\Delta \boldsymbol{a}=\left[\Delta u_{1}^{1}, \Delta u_{2}^{1}, \Delta u_{3}^{1}, \Delta u_{1}^{2}, \Delta u_{2}^{2}, \Delta u_{3}^{2}\right]^{\mathrm{T}}$ \\
\hline
\end{tabular}

Box C. 2

Expression of the different matrices involved in the secant stiffness matrix of the two node 3D truss element $(\alpha=1 / 2, \beta=0)$

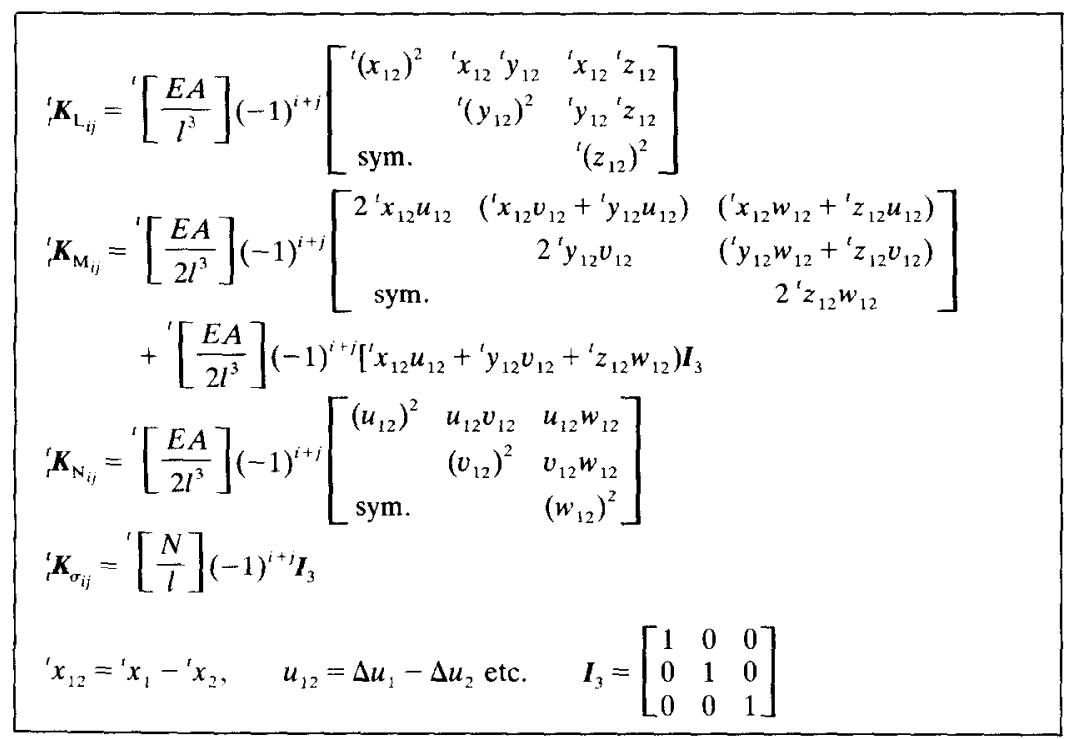

\section{References}

[1] J. Weinitschke, On the calculation of limit and bifurcation points in stability problems of elastic shells, Int. J. Solids Struct. 21 (1985) 1.

[2] R. Seydel, Numcrical computation of branch points in nonlinear structural analysis, Numer. Math. 33 (1979) $339-352$.

[3] P. Wriggers, W. Wagner and C. Miehe, A quadratically convergent procedure for the calculation of stability points in finite element analysis, Comput. Methods Appl. Mech. Engrg. 70 (1988) 329-347.

[4] P. Wriggers and J.C. Simo, A general procedure for the direct computation of turning and bifurcation points, Int. J. Numer. Methods Engrg. 30 (1990) 155-176.

[5] G. Skeie and C.A. Felippa, Detecting and traversing bifurcation points in nonlinear structural analysis, Int. J. Space Struct. 6(2) (1991) 77-98.

[6] W. Wagner, Calculation of bifurcation points via fold curves, in: P. Wriggers and W. Wagner, eds., Nonlinear Computational Mechanics, State-of-the-Art (Springer-Verlag, Berlin, 1991) 70-84. 
[7] Z. Waszczystzn, Numerical problems of nonlinear stability analysis of elastic structures, Comput. Struct. 17(1) (1983) $13-24$.

[8] E. Riks, Bifurcation and stability, a numerical approach, in: W.K. Liu, T. Belytschko and K.C. Park, eds., Proc. Int. Conf. on Innovative Methods for Nonlinear Problems (Pineridge Press International, 1984) 313-344.

[9] M.A. Fujikake, Simple approach to bifurcation and limit point calculations, Int. J. Numer. Methods Engrg. 21 (1985) 183-191.

[10] F. Nishino, W. Hartono, O. Fujiwara and P. Karasudhi, On tracing bifurcation equilibrium paths of geometrically nonlinear structures, Proc. of JSCE 380/1-7, April, Structural Engrg./Earthquake Engrg. (1987) 11s-17s.

[11] W. Wagner and P. Wriggers, A simple method for the calculation of postcritical branches, Engrg. Comput. 5 (1988) $103-109$.

[12] R. Kouhi and M. Mikkola, Tracing the equilibrium path beyond simple critical points, Int. J. Numer. Methods Engrg. 28 (1989) 2922-2941.

[13] E. Stein, W. Wagner and P. Wriggers, Nonlinear stability analysis of shell and contact-problems including branch-switching, Comput. Mech. 5 (1990) 428-446.

[14] H.A. Mang, On bounding properties of eigenvalues from linear initial FFC stability analysis of thin elastic shells with respect to stability limits from geometrically non-linear prebuckling analysis, Int. J. Numer. Methods Engrg. 32 (1991) $1087-1111$.

[15] P. Fuji, B.C. Perez and K.K. Choong, Selection of the control parameters in displacement incrementation, Comput. Struct. 42(2) (1992) 167-174.

[16] P. Fuji and K.K. Choong, Branch-switching in bifurcation problem of structures, J. Engrg. Mech., ASCE 118(8) (1992) $1578-1596$.

[17] E. Riks, An incremental approach to the solution of snapping and buckling problems, Int. J. Solids Struct. 15 (1979) 529-551 (1979).

[18] E. Riks, Some computational aspects of the stability analysis of nonlinear structures, Comput. Methods Appl. Mech. Engrg. 47 (1984) 219-259.

[19] K.K. Choong and Y. Hanagi, Review on methods of bifurcation analysis for geometrically non linear structures, Bulletin of IASS, 34 (1993) 133-149.

[20] E. Oñate, J. Oliver, J. Miquel and B. Suárez, A finite element formulation for geometrically non linear problems using a secant matrix, in: S. Atluri and G. Yagawa, eds., Proc. Int. Conf. on Computational Mechanics, May 25-29, Tokyo (Springer-Verlag, 1986).

[21] E. Onate, Possibilities of the secant stiffness matrix for non linear finite element analysis, in: N. Bicanic et al., eds., Non Linear Engineering Computations (Pineridge Press, 1991).

[22] E. Oñate, On the derivation and possibilities of the secant stiffness matrix for non linear finite element analysis, Comput. Mech. (1995) in press.

[23] H. Duddeck, B. Kroplin, D. Dinkler, J. Hillmann and W. Wagenhuber, Non linear computations in Civil Engineering Structures (in German), DFG Colloquium, 2-3 March 1989 (Springer-Verlag, Berlin, 1989).

[24] B. Kroplin, M. Wilhelm and M. Herrmann, Unstable phenomena in shect metal forming processes and their simulation, VDI. Berichte NR. 894, 1991, pp. 137-152.

[25] B. Kroplin, Instability prediction by energy perturbation, in: H. Alder, J.C. Heinrich, S. Lavanchy, E. Oñate and B. Suarez, eds., Numerical Methods in Applied Sciences and Engineering (CIMNE, Barcelona, 1992).

[26] J.M.T. Thompson and G.W. Hunt, A General Theory of Elastic Stability (John Wiley, 1973).

[27] M. Pignataro, N. Rizzi and A. Luongo, Stability, Bifurcation and Postcritical Elastic Structures (Elsevier, 1991).

[28] B. Kroplin, D. Dinkler and J. Hillmann, An energy perturbation method applied to non linear structural analysis, Comput. Methods Appl. Mech. Engrg. 52 (1985) 885-97.

[29] B. Kroplin and D. Dinkler, A material law for coupled local yielding and geometric instability, Engrg. Comput. 5(3) (1988) 210-216.

[30] B. Kroplin and D. Dinkler, Some thoughts on consistent tangent operators in plasticity in: D.R.J. Owen, E. Hinton and E. Oñate, eds., Computational Plasticity (Pineridge Press/CIMNE, 1990).

[31] E. Carrera, Sull' uso dell' operatore secante in analisi non lineare di strutture multistrato con il metodo degli elementi finiti, XI Congresso Nazionale AIMETA, Trento, 28 September-2 October, 1992.

[32] E. Carrera, On the application of the energy perturbation method to higher non linear problems, VIII Convegno Italiano di Meccanica Computazionale, AIMETA, Politecnico di Torino, 15-17 January, 1994, 149-254.

[33] R.H. Mal]et and P.V. Marcal, Finite element analysis of non-linear structures, J. Struct. Div., ASCE 14 (1968) 2081-2105.

[34] S. Rajasekaran and D.W. Murray, Incremental finite element matrices, J. Struct. Div., ASCE 99 (1973) $2423-2438$.

[35] C. Felippa, Discussions on Reference [34], J. Struct. Div., ASCE 100 (1974) 2519-2521.

[36] C. Felippa and L.A. Crivelli, The core-congruential formulation of geometrically non-linear TL finite elements, in: P. Wriggers and W. Wagner, eds., Non-Linear Computational Mechanics. The State-of-the-Art (Springer-Verlag, Berlin, 1991).

[37] C. Felippa, L.A. Crivelli and B. Haugen, A survey of the core-congruential formulation for geometrically non-linear TL finite elements, Arch. Comput. Methods Engrg. 1(1) (1994) 1-48.

[38] R.D. Wood and B. Schrefler, Geometrically non-linear analysis--A correlation of finite element notations, Int. J. Numer. Methods Engrg. 12 (1978) 635-642.

[39] M. Badawi and A.R. Cusens, Symmetry of the stiffness matrices for geometrically non linear analysis, Commun. Appl. Numer. Methods 8 (1992) 135-140.

[40] K.J. Bathe, Finite Element Procedures in Engineering Analysis (Prentice Hall, Englewood Cliffs, NJ, 1982).

[41] O.C. Zienkiewicz and R.L. Taylor, The Finite Element Method, McGraw-Hill, New York, Vol. I (1989) and Vol. II (1991).

[42] G.H. Powell and J. Simons, Improved iteration strategy for non-linear structures, Int. J. Numer. Methods Engrg. 17 (1981) 1455-1467. 
[43] M. Papadrakakis, Post-buckling analysis of spatial structures by vector iteration methods, Comput. Struct. 14(5-6) (1981) 393-402.

[44] R. Irles, Un modelo numérico para el análisis del colapso en entramados metálicos, Ph.D. Thesis (in Spanish), Universidad Politécnica de Valencia, 1985.

[45] H.H. Kowk, M.P. Kamat and L.T. Watson, Location of stable and unstable equilibrium configurations using a model truss region quasi-Newton method and tunnelling, Comput. Struct. 21 (1985) 909-916.

[46] K. Kondoh and S.N. Atluri, Influence of local buckling on global instability: simplified, large deformation, post-buckling analysis of plane trusses, Comput. Struct. 21(4) (1985) 613-627.

[47] M.A. Crisfield, Non Linear Finite Element Analysis of Solids and Structures, Vol. 1 (J. Wiley, New York, 1994). 